\title{
Heat flow, heat content and the isoparametric property *
}

\author{
Alessandro Savo
}

\begin{abstract}
Let $M$ be a Riemannian manifold and $\Omega$ a compact domain of $M$ with smooth boundary. We study the solution of the heat equation on $\Omega$ having constant unit initial conditions and Dirichlet boundary conditions. The purpose of this paper is to study the geometry of domains for which, at any fixed value of time, the normal derivative of the solution (heat flow) is a constant function on the boundary. We express this fact by saying that such domains have the constant flow property. In constant curvature spaces known examples of such domains are given by geodesic balls and, more generally, by domains whose boundary is connected and isoparametric. The question is: are they all like that?

In this paper we give an affirmative answer to this question: in fact we prove more generally that, if a domain in an analytic Riemannian manifold has the constant flow property, then every component of its boundary is an isoparametric hypersurface. For space forms, we also relate the order of vanishing of the heat content with fixed boundary data with the constancy of the $r$-mean curvatures of the boundary and with the isoparametric property. Finally, we discuss the constant flow property in relation to other well-known overdetermined problems involving the Laplace operator, like the Serrin problem or the Schiffer problem.
\end{abstract}

\section{Introduction}

In this paper, we prove a rigidity result for Riemannian manifolds with boundary satisfying a certain overdetermined problem for the heat equation; the aim is to understand the conditions on the heat content and the heat flow which insure the isoparametric property of the boundary. In this introduction, we first state the main results in Section 1.1 (Theorem 2 and Theorem 4); then, in Section 1.2, we relate the constant flow property to other overdetermined problems. The isoparametric property is recalled in Section 1.3 and, in Section 1.4, we state a general result valid in any smooth Riemannian manifold (Theorem 17). In Section 1.5, we show how Theorem 2 follows from Theorem 7 and finally, in Section 1.6, we give the plan of the paper with a rough scheme of the proofs.

\subsection{The constant flow property and the main results}

Let $M$ be a Riemannian manifold of dimension $n$, with metric tensor $g$, and let $\Omega$ be a compact domain in $M$ having smooth boundary $\partial \Omega$. A basic object in heat diffusion is

\footnotetext{
${ }^{*}$ Classification AMS 2000: 58J50, 35P15

Keywords: Heat equation, heat flow, overdetermined problems, isoparametric hypersurfaces
} 
the solution $u(t, x)$ of the heat equation on $\Omega$ with initial data 1 and Dirichlet boundary conditions:

$$
\left\{\begin{array}{l}
\Delta u+\frac{\partial u}{\partial t}=0 \quad \text { on } \Omega \\
u(0, x)=1 \text { for all } x \in \Omega \\
u(t, y)=0 \text { for all } y \in \partial \Omega \text { and } t>0
\end{array}\right.
$$

where $\Delta$ is the Laplace-Beltrami operator defined by the Riemannian metric $g$. We will often write $u(t, x)$ as $u_{t}(x)$ so that $u_{0}=1$. The interest in the function $u$ is also given by the fact that

$$
u(t, x)=\int_{\Omega} k(t, x, y) d y,
$$

where $k:(0, \infty) \times \Omega \times \Omega \rightarrow \mathbf{R}$ is the heat kernel of $\Omega$ (that is, the fundamental solution of the heat equation with Dirichlet boundary conditions). Note that $u(t, x)$ is the temperature at time $t$, at the point $x \in \Omega$, assuming that the initial temperature distribution is constant, equal to 1 , and that the boundary $\partial \Omega$ is kept at temperature zero at all times. Now let $\nu$ be the unit normal vector field of $\partial \Omega$, pointing inward, and let $y \in \partial \Omega$. Then, $\frac{\partial u}{\partial \nu}(t, y)$ can be interpreted as the heat flow at time $t$, at the boundary point $y$.

Definition 1. We say that $\Omega$ has the constant flow property if, for all fixed $t>0$, the heat flow

$$
\frac{\partial u}{\partial \nu}(t, \cdot): \partial \Omega \rightarrow \mathbf{R}
$$

is a constant function on $\partial \Omega$.

This property could be seen as an overdetermined problem for the heat equation. Overdetermined problems for the Laplacian have been vastly studied in the literature (see for example [3], [5], [6],[11], [13], [14], [15], [17], [19], [24], [29], [30]), and some of them will be recalled in Section 1.2 below. Solutions to a specific overdetermined problem exist only for special geometries, and in general one would like to classify all domains which support such solutions. For the constant flow property above we have the following rigidity theorem.

Theorem 2. Let $\Omega$ be a compact domain with smooth boundary in an analytic Riemannian manifold $M$. Assume that it has the constant flow property. Then each component of $\partial \Omega$ is an isoparametric hypersurface of $M$.

Theorem 2 follows from a more general result, valid in arbitrary smooth Riemannian manifolds (Theorem 7 below) and proved by studying the complete asymptotic expansion of the heat flow for small time, which was obtained in [21] and [22].

The definition and the main properties of isoparametric hypersurfaces will be recalled in Section 1.3 below; we only recall here that, in space forms, isoparametric hypersurfaces are characterized by having constant principal curvatures. When the ambient space is $\mathbf{R}^{n}$ or 
$\mathbf{H}^{n}$ the only compact isoparametric hypersurfaces are the geodesic spheres, and Theorem 2 asserts that in those cases the only domains with the constant flow property are geodesic balls (this is an easy case, immediately obtained from the Alexandrov theorem and the first two terms of the heat flow asymptotics, see the end of Section 3.1 for the short proof). Things get much more interesting and complicated in the sphere $\mathbf{S}^{n}$, also due to the fact that there is no analogue of the Alexandrov theorem, and that there is abundance of isoparametric hypersurfaces not isometric to geodesic spheres. From the work of Münzner [18] we know that a connected isoparametric hypersurface $\Sigma$ of the canonical sphere divides the sphere into two domains with common boundary $\Sigma$. It was proved by Shklover in ([25], Section 5.3 p. 562), that any spherical domain with connected, isoparametric boundary has the constant flow property. This fact, together with Theorem 2 above, gives the following characterization of the isoparametric property in space forms.

Corollary 3. A compact, connected, hypersurface of a space form is isoparametric if and only if it bounds a domain having the constant flow property.

Next, we examine the relations between the heat content and the isoparametric property. First, let us fix a smooth function $f \in C^{\infty}(\partial \Omega)$ and consider the solution $\tilde{f}_{t}(x) \doteq \tilde{f}(t, x)$ of the heat equation with zero initial temperature and with boundary temperature prescribed by the function $f$, that is:

$$
\left\{\begin{array}{l}
\Delta \tilde{f}_{t}+\frac{\partial \tilde{f}_{t}}{\partial t}=0 \\
\tilde{f}_{0}(x)=0 \text { for all } x \in \Omega \\
\tilde{f}_{t}(y)=f(y) \text { for all } y \in \partial \Omega \text { and } t>0 .
\end{array}\right.
$$

We call the function of $t \in(0, \infty)$ :

$$
\tilde{H}_{f}(t) \doteq \int_{\Omega} \tilde{f}_{t}
$$

the heat content with boundary data $f$. It is clear that $\lim _{t \rightarrow 0} \tilde{H}_{f}(t)=0$; if moreover $f$ integrates to zero on $\partial \Omega$ then $\tilde{H}_{f}(t)$ vanishes to order at least 1 as $t \rightarrow 0$. In fact, it is easy to prove (see Theorem 8 ) that

- A domain $\Omega$ has the constant flow property if and only if the heat content with boundary data in $C_{0}^{\infty}(\partial \Omega) \doteq\left\{f \in C^{\infty}(\partial \Omega): \int_{\partial \Omega} f=0\right\}$ is equal to zero at all times.

Then, domains with the constant flow property are perfect heat diffusers: the incoming heat, flowing inside from theregions where the boundary temperature is positive is perfectly balanced, at each time, by the outgoing heat, flowing away in correspondence to the negative boundary temperature: total heat content (44) is constant in time, hence always zero. This holds regardless of the temperature distribution on the boundary, as long as it has zero mean.

With this in mind, Theorem 2 asserts that if the heat flow with boundary data in $C_{0}^{\infty}(\partial \Omega)$ vanishes identically then each component of the boundary is isoparametric; that is, perfect 
heat diffusers have isoparametric boundary (and viceversa, at least in space forms, if the boundary is connected).

In our second main result, we observe that the order of vanishing of the heat content with boundary data in $C_{0}^{\infty}(\partial \Omega)$ is somewhat related to a kind of degree of isoparametricity of the boundary, at least if the ambient space has constant curvature. Moreover, we will also prove that only a certain (finite) order of vanishing is needed in order to insure the isoparametric property: in a certain sense we can weaken, in space forms, the constant flow assumption of Theorem 2 .

To be more precise, let $S$ denote the shape operator $\partial \Omega$, with eigenvalues (i.e. principal curvatures) denoted $k_{1}, \ldots, k_{n-1}$. For $r=1, \ldots, n-1$, define the mean curvature of order $r$ as the $r$-th elementary function of the principal curvatures:

$$
E_{r}=\sum_{1 \leq i_{1}<\cdots<i_{r} \leq n-1} k_{i_{1}} \ldots k_{i_{r}}
$$

(other authors normalize by a suitable constant, but this would not affect the discussion here). For $r=1$ we get indeed a multiple of the mean curvature, and for $r=n-1$ the Gauss-Kronecker curvature. In fact, $E_{r}$ is the coefficient of $x^{n-1-r}$ in the characteristic polynomial of $S$ (up to sign). Our second main result is the following. As usual, the writing $\tilde{H}_{f}(t) \sim o\left(t^{\alpha}\right)$ means that $\lim _{t \rightarrow 0} t^{-\alpha} \tilde{H}_{f}(t)=0$. For the proof, see Section 4.2 ,

\section{Theorem 4.}

(a) Let $\Omega$ be a domain in $\mathbf{R}^{n}$ or $\mathbf{H}^{n}$. If $\tilde{H}_{f}(t) \sim o(t)$ for all $f \in C_{0}^{\infty}(\partial \Omega)$, then $\Omega$ is a geodesic ball.

(b) Now assume that $\Omega$ is a domain in $\mathbf{S}^{n}$, and that, for some integer $k \geq 2, \tilde{H}_{f}(t) \sim o\left(t^{\frac{k}{2}}\right)$ for all $f \in C_{0}^{\infty}(\partial \Omega)$. Then the mean curvatures $E_{1}, \ldots, E_{k-1}$ are all constant on $\partial \Omega$.

(c) In particular, if $\Omega \subseteq \mathbf{S}^{n}$ and $\tilde{H}_{f}(t) \sim o\left(t^{\frac{n}{2}}\right)$ for all $f \in C_{0}^{\infty}(\partial \Omega)$, then $\partial \Omega$ is isoparametric (and consequently $\tilde{H}_{f}(t)$ is identically zero at all times).

Again, the different behavior in (a) (which is very easy to prove) as opposed to (b) and (c) (which are much more complicated) is due essentially to the validity of the Alexandrov theorem.

\subsection{Relation with other overdetermined problems}

Perhaps the seminal work in this field was done by J. Serrin, who considered the following overdetermined problem, and inspired a good part of the following research:

$$
\left\{\begin{array}{l}
\Delta v=1 \quad \text { on } \quad \Omega, \\
v=0, \quad \frac{\partial v}{\partial \nu}=c \quad \text { on } \quad \partial \Omega,
\end{array}\right.
$$

where $c$ is a constant. Problem (5) is often referred to as the Serrin problem. Domains supporting a solution to (5) are termed harmonic in [20] because they are characterized by 
the following property: the mean value of a harmonic function on $\Omega$ equals its mean value on $\partial \Omega$. Serrin's celebrated result states that the only Euclidean domains which support a solution to (5) are balls. More generally, he proved in 24] that the only Euclidean domains admitting a positive solution to the overdetermined problem

$$
\left\{\begin{array}{l}
\Delta v=F(v) \quad \text { on } \quad \Omega, \\
v=0, \quad \frac{\partial v}{\partial \nu}=c \quad \text { on } \quad \partial \Omega,
\end{array}\right.
$$

are balls. This rigidity result was later extended to the hyperbolic space and the hemisphere in [14]. However, on the whole sphere (and in any other manifold) the classification problem is, to the best of our knowledge, still open (but see the next Section).

Another famous problem is the so-called Schiffer problem (D) for a given Dirichlet eigenvalue $\lambda$ (for more details see [25]):

$$
\left\{\begin{array}{l}
\Delta u=\lambda u \text { on } \Omega, \\
u=0, \frac{\partial u}{\partial \nu}=c \quad \text { on } \quad \partial \Omega .
\end{array}\right.
$$

We note that on a Euclidean ball any radial eigenfunction is a solution to (77); thus, there are solutions to the above problem for infinitely many eigenvalues $\lambda$. The following conjecture, proposed by Berenstein and often called Schiffer conjecture (D), seems to be still open, even in Euclidean space:

Conjecture. Let $\Omega$ be a Euclidean domain and let $\lambda$ be any fixed Dirichlet eigenvalue of $\Omega$. If $\Omega$ supports a solution to (7) then $\Omega$ is a ball.

There are several partial results related to this conjecture, see for example [6]. It is also known that the Neumann version of this conjecture (obtained by changing the boundary conditions in (7) to $\frac{\partial u}{\partial \nu}=0, u=c$ and known as Schiffer conjecture $\left.(N)\right)$ is equivalent to the famous Pompeiu problem (the interested reader could consult [5] and [30]).

Now let us consider problem (7) when $\lambda=\lambda_{1}(\Omega)$, the lowest Dirichlet eigenvalue of $\Omega$ :

$$
\left\{\begin{array}{l}
\Delta u=\lambda_{1}(\Omega) u \quad \text { on } \quad \Omega, \\
u=0, \frac{\partial u}{\partial \nu}=c \quad \text { on } \quad \partial \Omega .
\end{array}\right.
$$

In this case, domains for which a solution exists are called extremal domains: they are critical points of the first Dirichlet eigenvalue under volume preserving deformations of $\Omega$ (this follows from Hadamard's formula, see [23] and [11]). It follows immediately from Serrin's result [24], and the existence of a positive eigenfunction associated to $\lambda_{1}(\Omega)$, that the only Euclidean domains supporting a solution to (8) are balls: this by the way shows that the Schiffer conjecture (D) above is true for the first eigenvalue. However, the classification of extremal domains in general Riemannian manifolds (in particular, in the sphere) is still an open problem. Interesting families of extremal domains of small 
prescribed volume are shown to exist near any nondegenerate critical point of the scalar curvature of any Riemannian manifold: see [19].

We will observe in Section 2 that the constant flow property implies existence of a solution to all of the above problems (see also [25]). That is:

Theorem 5. Let $\Omega$ be a Riemannian domain having the constant flow property. Then:

a) $\Omega$ supports a solution to the Serrin problem (5);

b) $\Omega$ supports a solution to the Schiffer problem (7) for infinitely many Dirichlet eigenvalues;

c) $\Omega$ is an extremal domain (that is, it supports a solution to (8)).

For the proof, see Section 2.

Finally let us mention the results of Magnanini and Sakaguchi in [16, related to the function $u(t, x)$ defined in (11). A hypersurface $\Sigma$ contained in the interior of $\Omega$ is said to be stationary isothermic if it is isothermic for all times $t>0$; that is, if there exists a smooth function $\psi:(0, \infty) \rightarrow(0, \infty)$ such that:

$$
u(t, x)=\psi(t) \text { for all } t>0 \text { and } x \in \Sigma .
$$

The authors then show that if $\Omega$ is a bounded, convex domain of $\mathbf{R}^{n}$ admitting a stationary isothermic hypersurface, then $\Omega$ is a ball (the result continues to hold under less restrictive assumptions on the boundary of $\Omega$, see [16] for more details). This problem could also be seen as an overdetermined problem for the heat equation.

\subsection{The isoparametric property}

We have seen that classification theorems for the Serrin problem (5) and for extremal domains have been proved so far only when the ambient manifold is Euclidean space, the hyperbolic space or the hemisphere: therefore, the natural question is whether, on the whole sphere, there exist other "exotic" examples (that is, examples not isometric to geodesic balls).

The answer is actually affirmative, and a first family of such examples was constructed by Berenstein in [4]: this is the family of domains in $\mathbf{S}^{n}$ bounded by certain Clifford tori (tubes around a great circle in $\mathbf{S}^{n}$ ). In fact, these domains admit solutions to the Schiffer problem (7) for infinitely many eigenvalues.

The matter was later expanded and clarified in [25], where it is observed that if a domain in $\mathbf{S}^{n}$ has a connected, isoparametric boundary then it supports a solution to the Serrin problem and also to the Schiffer problem $(D)$ for infinitely many eigenvalues (see [25], Theorem 2 p. 549). Moreover, it has the constant flow property in the sense of Definition 1 (as proved in Section 5.3 of [25]). Thus, the sphere hosts a large variety of new examples. Let us then recall the general definition of the isoparametric property. 
Let $M$ be a Riemannian manifold and $U$ an open subset of $M$. A smooth function $F: U \rightarrow \mathbf{R}$ is called isoparametric if there exist smooth functions $A$ and $B$ defined on the range of $F$ such that:

$$
\left\{\begin{array}{l}
\Delta F=A \circ F, \\
|\nabla F|^{2}=B \circ F .
\end{array}\right.
$$

Then, the (smooth) hypersurface $\Sigma$ of $U \subseteq M$ is called isoparametric if it is a regular level set of an isoparametric function. In fact, any isoparametric function defines a whole one-parameter family of isoparametric hypersurfaces, and any two members of the family are at constant distance to each other. For the main facts on isoparametricity see the standard reference [27] and also [28]. Let us observe some equivalent, more geometric, definitions. If $\Sigma$ is a smooth hypersurface of $M$ and $\rho: M \rightarrow \mathbf{R}$ is the distance function to $\Sigma$, then the level sets (equidistants) $\rho^{-1}(r)$ are smooth provided that $r<\epsilon$ is small enough. We have the following characterizations; the first follows easily from the definition (9), while the second is due to Cartan [9].

\section{Theorem 6.}

a) The hypersurface $\Sigma$ is isoparametric if and only if all equidistants sufficiently close to $\Sigma$ have constant mean curvature.

b) $\Sigma$ is isoparametric in a space form $M$ if and only if it has constant principal curvatures (that is, the characteristic polynomial of the shape operator of $\Sigma$ is the same at all points).

As proved by Cartan, the only compact isoparametric hypersurfaces of Euclidean or hyperbolic space are geodesic balls; on the other hand, on the sphere the situation is much more interesting, and has generated deep mathematical research, starting from Cartan himself. After the work of Münzner [18] we know that isoparametric hypersurfaces of the sphere are given by level sets of restrictions to $\mathbf{S}^{n}$ of certain (globally defined) homogeneous polynomials in $\mathbf{R}^{n+1}$, called Cartan-Münzner polynomials, and that the number $g$ of distinct principal curvatures can be only $g=1,2,3,4$ and 6 . Families of isoparametric hypersurfaces have been constructed in each of the above cases: for example, Clifford tori correspond to $g=2$ and, for $g=4$, there exist examples with non-homogeneous boundary.

Let us briefly explain why a spherical domain $\Omega$ bounded by a connected isoparametric boundary $\Sigma$ has the constant flow property. From the general theory one knows that $\Omega$ is a smooth tube of constant radius around a smooth (minimal) submanifold $N$ of codimension at least two (the focal variety of $\Sigma$ ). The crucial fact is that the equidistants from $N$ (hence, also the equidistants from $\partial \Omega=\Sigma$ ) all have constant mean curvature. Now, if one defines radial functions as those functions which are constant on the equidistants, then one can verify that the Laplacian of $\Omega$ takes radial functions to radial functions. In turn, this implies that the solution of the heat equation with radial initial data (in particular, our function $u_{t}$ ) will stay radial at all times: as a consequence, its normal derivative $\partial u_{t} / \partial \nu$ (the heat flow) will be constant on the boundary for all times. Then, any such domain has the constant flow property. 
The approach followed in [25], Section 5.3 to prove this fact is to work directly with definition (91) and to use the Fourier series representation of $u(t, x)$ (see equation (5.8)).

We conclude the section by asking whether the existence of a solution to the Serrin problem, or to the other overdetermined problems examined above, in spaces different from the Euclidean space, the hyperbolic space or the hemisphere (where the answer is known) would imply some kind of isoparametric property of the boundary. Also, is it true that any domain admitting a solution to the Serrin problem must also have the constant flow property? This would be a converse to Theorem 5. There is no immediate reason to have a positive answer; however we don't have, at the moment, any specific counterexample.

\subsection{A general theorem on Riemannian manifolds}

Theorem 2 will follow from a more general result: Theorem 7 below. Let then $\Omega$ be a compact domain with smooth boundary in a Riemannian manifold $M$ and let $\rho: \Omega \rightarrow \mathbf{R}$ now denote the distance function to the boundary of $\Omega: \rho(x)=\operatorname{dist}(x, \partial \Omega)$. As the boundary is smooth, there exist a small $\epsilon>0$ such that the function $\rho$ will be smooth in the $\epsilon$-tubular neighborhood $U$ of $\partial \Omega$ :

$$
U=\{x \in \Omega: 0 \leq \rho(x)<\epsilon\}
$$

(precisely, when $\epsilon$ is small enough so that $U$ does not meet the cut-locus of the normal exponential map). It is also well-known (and easy to verify) that, at each point $x \in U$ at distance $\rho(x)=r$ to the boundary, the level set $\rho^{-1}(r)$ is smooth and one has:

$$
\Delta \rho(x)=\text { trace of the second fundamental form of the equidistant } \rho^{-1}(r) .
$$

In other words, $\Delta \rho$ measures the mean curvature of the interior parallels (equidistants). Let us write, for simplicity:

$$
\eta \doteq \Delta \rho
$$

thus obtaining a smooth function on $U$. Note that the vector field $\nu=\nabla \rho$ is smooth on $U$ and is everywhere normal to the level sets $\rho^{-1}(r)$; when restricted on $\partial \Omega$, it will give the inner unit normal field.

Theorem 7. Let $\Omega$ be domain with smooth boundary in a Riemannian manifold $M$. Assume that $\Omega$ has the constant flow property. Then $\frac{\partial^{k} \eta}{\partial \nu^{k}}$ is constant on $\partial \Omega$ for all $k=0,1,2, \ldots$

The proof will be done in Section 3. Note that the result holds in any smooth (not necessarily analytic) Riemannian manifold. We can now show how Theorem 2 follows easily from Theorem 7 . 


\subsection{Proof of Theorem 2}

By assumption, the ambient manifold $M$ is analytic. By the regularity results in [13] and [31] we know that, if a domain admits a solution to the Schiffer problem (7) for some eigenvalue $\lambda$, then its boundary must be analytic. As proved in Theorem 5 , any domain having the constant flow property admits a solution to the Schiffer problem (for infinitely many eigenvalues). Then, $\partial \Omega$ is analytic.

Consider the tubular neighborhood $U=\{\rho<\epsilon\}$ of $\partial \Omega$ as in (10). We will show that the nearby equidistants $\rho^{-1}(r)$ have constant mean curvature for all $r<\epsilon$, or, equivalently, that $\eta$ is constant on $\rho^{-1}(r)$ for $r<\epsilon$. Consider the diffeomorphism:

$$
\Phi:[0, \epsilon) \times \partial \Omega \rightarrow U
$$

defined by $\Phi(r, y)=\exp _{y}(r \nu(y))$, where $\nu(y)$ is the inner unit normal at $y \in \partial \Omega$. The pair $(r, y)$ gives rise to the normal coordinates of a point of $U$. As both $M$ and $\partial \Omega$ are analytic, the normal exponential map, hence also the map $\Phi$, must be analytic. Now, the composition of $\Phi^{-1}$ with the projection onto $[0, \epsilon)$, which is precisely the distance function $\rho$ on $U$, is also analytic, hence its Laplacian (the function $\eta$ ), is analytic. Let $x \in U$ be a point at distance $r$ to the boundary, and let $y$ be the foot of the minimizing geodesic segment from $x$ to $\partial \Omega$, so that $(r, y)$ are the normal coordinates of $x$. As $\eta$ is analytic, $\eta(x)=\eta(r, y)$ equals the sum of its $r$-Taylor series based at $(0, y)$. Hence:

$$
\eta(x)=\sum_{k=0}^{\infty} \frac{1}{k !} \frac{\partial^{k} \eta}{\partial \nu^{k}}(y) r^{k} .
$$

By Theorem [7, one has $\frac{\partial^{k} \eta}{\partial \nu^{k}}(y)=a_{k}$ for all $k$, where $a_{k}$ is independent on $y$; this shows that the right-hand side of the previous equation does not depend on $y$, but only on $r=\rho(x)$ : hence $\eta$ is constant on $\rho^{-1}(r)$ and $\partial \Omega$ is isoparametric.

\subsection{Plan of the paper and scheme of the proof}

- In Section 2 we first show that the constant flow property is equivalent to a vanishing condition for the heat content function with (zero mean) boundary data. We then verify that a domain with the constant flow property supports a solution to the Serrin problem (Theorem 91), and also a solution to the Schiffer problem for infinitely many eigenvalues (Theorem 10).

- In Section 3 we start the proof of Theorem 7 , by recalling the main results on the asymptotic expansion of the heat content and the heat flow proved in [21] and [22]. These results will be needed for the proof of Theorem 7 . In fact, it turns out that the heat flow at a point $y \in \partial \Omega$ admits a complete asymptotic series, as $t \rightarrow 0$, of the following type:

$$
\frac{\partial u}{\partial \nu}(t, y) \sim \frac{1}{\sqrt{\pi}} \cdot \frac{1}{\sqrt{t}}+\sum_{k=0}^{\infty}\left(1+\frac{k}{2}\right) B_{k+2}(y) \cdot t^{k / 2}
$$


for a sequence of smooth invariants $B_{k}(y) \in C^{\infty}(\partial \Omega)$. Clearly, if $\Omega$ has the constant flow property then all these invariants must be constant functions on $\partial \Omega$ : from this information, after some work, one can eventually derive that the normal derivative, of arbitrary order, of the function $\eta$ is constant on the boundary.

More in detail, it follows from the results in [22] that the above invariants can be written as $B_{k}=-\bar{D}_{k} \eta$; here $\bar{D}_{k}$ is a certain differential operator belonging to the algebra generated by the Laplacian $\Delta$ of $\Omega$ and by the operator $N$ acting on $\phi \in C^{\infty}(U)$ as follows:

$$
N \phi=2\langle\nabla \phi, \nabla \rho\rangle-\phi \Delta \rho=2 \frac{\partial \phi}{\partial \nu}-\eta \phi
$$

where $\rho$ is the distance function to $\partial \Omega$ and where we have set $\nu=\nabla \rho$. The operator $\bar{D}_{k}$ can be computed using an explicit recursive scheme defining some related operators $D_{k}$ : this scheme was proved in 21] and will be recalled in Theorem 11. The proof of Theorem 7 will be by induction on the order $k$ of the normal derivative of $\eta$, and in Section 3.1 we illustrate the general strategy by proving Theorem 7 for $k \leq 2$.

- In Section 4 we prove Theorem 2 and Theorem 4. We first prove Proposition 15, in which we relate the invariants $B_{k}$ in (11) with the normal derivatives of $\eta$. More precisely we prove that, if the function $\eta$ restricts to a constant function on $\partial \Omega$ together with all of its normal derivatives up and including the order $k$ then, for all $y \in \partial \Omega$ one has:

$$
B_{k+3}(y)=-w\left(\bar{D}_{k+3}\right) \frac{\partial^{k+1} \eta}{\partial \nu^{k+1}}(y)+b_{k}
$$

where $b_{k}$ is a constant which does not depend on $y \in \partial \Omega$. Here $w\left(\bar{D}_{k+3}\right)$ (the so-called weight of the operator $\left.\bar{D}_{k+3}\right)$ is the coefficient of the highest order normal derivative in $\bar{D}_{k+3}$. Assume that $w\left(\bar{D}_{k+3}\right)$ is non-zero for all $k$. Then, an easy inductive argument (Proposition 17) shows that, if $B_{2}, \ldots, B_{m}$ are constant on $\partial \Omega$ for some $m \geq 2$, then the functions $\eta, \frac{\partial \eta}{\partial \nu}, \ldots, \frac{\partial^{m-2} \eta}{\partial \nu^{m-2}}$ will also be constant on $\partial \Omega$. The proof of Theorem 7 now follows immediately, while the proof of Theorem 4 requires an additional argument involving the Newton identities (see Section 4.2).

In conclusion, all the results of the paper will be completely proved once we show that $w\left(\bar{D}_{k+3}\right)$ is non-zero for all $k$; thus in Theorem [16] of Section 4 we state the main combinatorial result, giving the explicit expression of the weight of $\bar{D}_{k}$ for all $k$.

- In the remaining sections we prove Theorem 16, by applying the recursive scheme which define the operators $\bar{D}_{k}$. For convenience, we have divided the proof in several sections. The proof is progressively reduced to a set of combinatorial identities for the so-called Hankel transforms associated to a certain numerical sequence (see Lemma 24); these identities can be derived from the work of Tamm in [26], and we give an explicit account of that in the Appendix (Section 9). 


\section{Equivalent condition and other overdetermined problems}

Given a smooth function $\phi(x)$ on $\Omega$, we let $\phi_{t}(x) \doteq \phi(t, x)$ be the solution of the heat equation with initial data $\phi_{0}(x)=\phi(x)$ and Dirichlet boundary conditions:

$$
\left\{\begin{array}{l}
\Delta \phi_{t}+\frac{\partial \phi_{t}}{\partial t}=0 \\
\phi_{0}=\phi \text { on } \Omega \\
\phi_{t}=0 \text { on } \partial \Omega, \text { for all } t>0 .
\end{array}\right.
$$

The total heat inside the domain at time $t$ is measured by the heat content function, defined for $t \geq 0$ by:

$$
H_{\phi}(t)=\int_{\Omega} \phi_{t}
$$

$H_{\phi}(t)$ is smooth for $t>0$ but only continuous at $t=0$; it will also be called the heat content with initial data $\phi$. In what follows, we consider the following spaces:

$$
C_{0}^{\infty}(\partial \Omega)=\left\{f \in C^{\infty}(\partial \Omega): \int_{\partial \Omega} f=0\right\}, \quad \mathcal{H}_{0}(\Omega)=\left\{\phi \in C^{\infty}(\Omega): \Delta \phi=0, \int_{\partial \Omega} \phi=0\right\} .
$$

Recall that, given $f \in C^{\infty}(\partial \Omega)$ we denoted by $\tilde{f}_{t}$ the solution of the heat equation with zero initial conditions and boundary conditions prescribed by $f$ : see (3). It is clear that, if $\phi$ is the harmonic extension of $f$ to $\Omega$ (that is, if $\phi$ satisfies $\Delta \phi=0$ on $\Omega$ and $\phi=f$ on $\partial \Omega$ ) then $\tilde{f}_{t}$ can be written:

$$
\tilde{f}_{t}=\phi-\phi_{t}
$$

for all $t>0$. Integrating on $\Omega$, we see that the heat content with boundary data $f$, that is, the function $\tilde{H}_{f}(t)=\int_{\Omega} \tilde{f}_{t}$, can be written:

$$
\tilde{H}_{f}(t)=\int_{\Omega} \phi-H_{\phi}(t)
$$

where $\phi$ is the harmonic extension of $f$ to $\Omega$.

We will often use the fact that the only functions on $\partial \Omega$ which are $L^{2}$-orthogonal to $C_{0}^{\infty}(\partial \Omega)$ are the constants.

Theorem 8. A domain $\Omega$ has the constant flow property if and only if:

(a) the heat content with initial data in $\mathcal{H}_{0}(\Omega)$ is identically zero at all times; that is, one has $H_{\phi}(t)=0$ for all $\phi \in \mathcal{H}_{0}(\Omega)$ and for all $t \geq 0$.

(b) the heat content with boundary data in $C_{0}^{\infty}(\partial \Omega)$ is identically zero at all times; that is, one has $\tilde{H}_{f}(t)=0$ for all $f \in C_{0}^{\infty}(\partial \Omega)$ and for all $t \geq 0$. 
Proof. (a) We first observe that we have the identity, valid for all $t>0$ :

$$
H_{\phi}(t)=\int_{\Omega} \phi u_{t}
$$

where $u_{t}$ is, as usual, the solution of our original equation (1). In fact, if $k(t, x, y)$ denotes the heat kernel of $\Omega$ with Dirichlet boundary conditions, one has $\phi_{t}(x)=\int_{\Omega} k(t, x, y) \phi(y) d y$ hence:

$$
\int_{\Omega} \phi_{t}(x) d x=\int_{\Omega}\left(\int_{\Omega} k(t, x, y) \phi(y) d y\right) d x=\int_{\Omega} \phi(y)\left(\int_{\Omega} k(t, x, y) d x\right) d y=\int_{\Omega} \phi(y) u_{t}(y) d y .
$$

If $\phi$ is harmonic on $\Omega$ we obtain, from (14), the Green formula and the fact that $u_{t}$ vanishes on the boundary:

$$
H_{\phi}^{\prime}(t)=-\int_{\Omega} \phi \Delta u_{t}=-\int_{\partial \Omega} \phi \frac{\partial u_{t}}{\partial \nu} .
$$

Now assume that $\phi \in \mathcal{H}_{0}(\Omega)$ : then $\phi$ is harmonic and $\int_{\partial \Omega} \phi=0$. If $\Omega$ has the constant flow property then $\frac{\partial u_{t}}{\partial \nu}$ is constant on $\partial \Omega$, hence it can be taken out of the integral (15) so that $H_{\phi}^{\prime}(t)=0$ for all $t>0$. Therefore, for all $t$ :

$$
H_{\phi}(t)=H_{\phi}(0)=\int_{\Omega} \phi
$$

Now, since $\phi_{t}$ converges uniformly to zero as $t \rightarrow \infty$, so does $H_{\phi}(t)$, which in turn implies that $\int_{\Omega} \phi=0$ : hence $H_{\phi}(t)=0$ for all $t$.

Conversely, assume that $H_{\phi}(t)=0$ for all $t \geq 0$ and $\phi \in \mathcal{H}_{0}(\Omega)$. It is enough to show that

$$
\int_{\partial \Omega} f \frac{\partial u_{t}}{\partial \nu}=0
$$

for all $f \in C_{0}^{\infty}(\partial \Omega)$ and $t>0$, because then $\frac{\partial u_{t}}{\partial \nu}$ must be constant on $\partial \Omega$. Fix $f \in C_{0}^{\infty}(\partial \Omega)$ and consider the unique harmonic function $\phi$ which extends $f$ to $\Omega$. By assumption $\phi \in \mathcal{H}_{0}(\Omega)$ hence $H_{\phi}(t)=0$ for all $t$. But then $H_{\phi}^{\prime}(t)=0$ for all $t$ and by (15) we see

$$
0=-\int_{\partial \Omega} \phi \frac{\partial u_{t}}{\partial \nu}=-\int_{\partial \Omega} f \frac{\partial u_{t}}{\partial \nu}
$$

hence the assertion.

Proof of (b). It follows immediately from (a) and (13). It can also be proved by observing that, if $f \in C_{0}^{\infty}(\partial \Omega)$ and if $\phi$ is its harmonic extension to $\Omega$, then, by (13) and (15):

$$
\tilde{H}_{f}^{\prime}(t)=\int_{\partial \Omega} f \frac{\partial u_{t}}{\partial \nu}
$$

One can then argue as before. 
We can now show the following fact.

Theorem 9. Any Riemannian domain $\Omega$ having the constant flow property supports a solution to the Serrin problem (5).

Proof. Assume that $\Omega$ has the constant flow property and let $v$ be the unique function such that $\Delta v=1$ on $\Omega$ and $v=0$ on the boundary. We have to show that its normal derivative is constant on $\partial \Omega$. Fix $f \in C_{0}^{\infty}(\partial \Omega)$ and extend $f$ to a harmonic function $\phi$ on $\Omega$. Then, by Theorem 8 we know that the heat content with initial data $\phi$ is identically zero: $\int_{\Omega} \phi_{t}=0$ for all $t \geq 0$. Taking $t=0$ we see that $\phi$ integrates to zero on $\Omega$. Therefore, by the Green formula:

$$
0=\int_{\Omega} \phi=\int_{\Omega} \phi \Delta v=\int_{\partial \Omega} \phi \frac{\partial v}{\partial \nu}=\int_{\partial \Omega} f \frac{\partial v}{\partial \nu}
$$

As $f \in C_{0}^{\infty}(\partial \Omega)$ was arbitrary, one must have $\frac{\partial v}{\partial \nu}=$ const.

Theorem 10. Let $\Omega$ be a domain with the constant flow property. Then the overdetermined problem

$$
\left\{\begin{array}{l}
\Delta u=\lambda u \text { on } \quad \Omega \\
u=0, \frac{\partial u}{\partial \nu}=c \quad \text { on } \quad \partial \Omega
\end{array}\right.
$$

admits a solution for an infinite sequence $\left\{\lambda_{j}^{+}\right\}$of eigenvalues, in particular, for $\lambda=$ $\lambda_{1}(\Omega)$. Hence, any domain with the constant flow property is also extremal.

Proof. Let $\operatorname{Spec}(\Omega)=\left\{\lambda_{1}, \lambda_{2}, \ldots\right\}$ be the spectrum of $\Omega$ for the Dirichlet boundary conditions. We single out an (infinite) $\operatorname{subset}_{\operatorname{Spec}}(\Omega) \subseteq \operatorname{Spec}(\Omega)$, as follows:

$\operatorname{Spec}_{+}(\Omega)=\left\{\lambda \in \operatorname{Spec}(\Omega):\right.$ there exists an eigenfunction $\phi \in V(\lambda)$ such that $\left.\int_{\Omega} \phi \neq 0\right\}$.

As any eigenfunction associated to $\lambda_{1}$ does not change sign, we have that $\lambda_{1} \in \operatorname{Spec}_{+}(\Omega)$. The aim is to prove that if a domain $\Omega$ has the constant flow property then the problem at hand (Schiffer problem (D)) admits a solution for all $\lambda \in \operatorname{Spec}_{+}(\Omega)$. Let us list the elements of $\operatorname{Spec}_{+}(\Omega)$ (in increasing order) as follows:

$$
\operatorname{Spec}_{+}(\Omega)=\left\{\lambda_{1}^{+}, \lambda_{2}^{+}, \ldots\right\}
$$

Note that $\lambda_{1}^{+}=\lambda_{1}$. Let us briefly justify why this subset is actually infinite. Writing the Fourier series expansion of the constant function 1, one sees that only the eigenvalues in $\operatorname{Spec}_{+}(\Omega)$ contribute with a non-zero term: if this set were finite then the Fourier series would also be finite, which is impossible because otherwise one would have $1=0$ on the boundary. 
We now construct a special orthonormal basis of eigenfunctions. Given $\lambda_{j}^{+} \in \operatorname{Spec}_{+}(\Omega)$ consider the linear map $I: V\left(\lambda_{j}^{+}\right) \rightarrow \mathbf{R}$ given by integration over $\Omega$ :

$$
I \phi=\int_{\Omega} \phi
$$

As the orthogonal complement of the kernel of $I$ is one-dimensional, we can select an orthonormal basis of $V\left(\lambda_{j}\right)$ as follows:

$$
\left\{\phi_{j}^{+}, \psi_{1}, \ldots, \psi_{m}\right\}
$$

where $\phi_{j}^{+}$has a positive integral over $\Omega$, while all the other eigenfunctions $\psi_{j}$ (if $m \geq 1$ ) have zero integral. We do this for every element in $\operatorname{Spec}_{+}(\Omega)$; for the eigenvalues which do not belong to $\operatorname{Spec}_{+}(\Omega)$, we take any orthonormal basis of the respective eigenspace. Repeating the procedure for all eigenvalues, we obtain a special orthonormal basis of $L^{2}(\Omega)$.

Let $\operatorname{Spec}(\Omega)=\left\{\lambda_{1}, \lambda_{2}, \ldots\right\}$ be the full Dirichlet spectrum of $\Omega$ (eigenvalues are repeated according to multiplicity), and select any othonormal basis of associated eigenfunctions $\left\{\phi_{j}\right\}_{j=1,2, \ldots}$. Then, the heat kernel for the Dirichlet conditions is:

$$
k(t, x, y)=\sum_{j=1}^{\infty} e^{-\lambda_{j} t} \phi_{j}(x) \phi_{j}(y),
$$

and the heat content with initial data $f$ has the following Fourier expansion:

$$
H_{f}(t)=\sum_{j=1}^{\infty} e^{-\lambda_{j} t} \int_{\Omega} \phi_{j} \cdot \int_{\Omega} f \phi_{j} .
$$

We now adopt the special orthonormal basis constructed above: it is clear that only the eigenvalues in $\operatorname{Spec}_{+}(\Omega)$ show up

$$
H_{f}(t)=\sum_{j=1}^{\infty} e^{-\lambda_{j}^{+} t} \int_{\Omega} \phi_{j}^{+} \cdot \int_{\Omega} f \phi_{j}^{+}
$$

and that there is only one term containing each $\lambda_{j}^{+} \in \operatorname{Spec}_{+}(\Omega)$.

We can now prove the Theorem. First assume that $\Omega$ has the constant flow property and fix $\psi \in \mathcal{H}_{0}(\Omega)$. From the previous theorem, we know that $H_{\psi}(t)=0$ for all $t$. From (17) we easily get (since there is only one term corresponding to $\lambda_{j}^{+}$):

$$
\int_{\Omega} \phi_{j}^{+} \cdot \int_{\Omega} \psi \phi_{j}^{+}=0
$$

for all $j$ and in turn

$$
\int_{\Omega} \psi \phi_{j}^{+}=0
$$


for all $j$ because $\int_{\Omega} \phi_{j}^{+}$is positive by definition. Fix $j$. As $\psi$ is harmonic on $\Omega$ we see, by the Green formula:

$$
\int_{\partial \Omega} \psi \frac{\partial \phi_{j}^{+}}{\partial \nu}=\lambda_{j}^{+} \int_{\Omega} \psi \phi_{j}^{+}=0
$$

The above holds for all $\psi \in \mathcal{H}_{0}(\Omega)$; as any element of $C_{0}^{\infty}(\partial \Omega)$ is the restriction to the boundary of an element of $\mathcal{H}_{0}(\Omega)$, the above immediately implies that $\frac{\partial \phi_{j}^{+}}{\partial \nu}$ is constant on $\partial \Omega$ which proves that the pair $\left(\lambda_{j}^{+}, \phi_{j}^{+}\right)$is a solution of the Schiffer problem (D).

More precisely, we have proved that a domain $\Omega$ has the constant flow property if and only if the pair $\left(\lambda_{j}^{+}, \phi_{j}^{+}\right)$is a solution of the Schiffer problem (D) for all $\lambda_{j}^{+} \in \operatorname{Spec}_{+}(\Omega)$.

\section{Asymptotics of the heat flow: review}

In this section we will review the main results on the asymptotics of the heat flow, which were proven in [21] and [22], and which will be used in this paper.

Given a smooth function $\phi \in C^{\infty}(\Omega)$, we let $\phi_{t}(x)$ be the solution of the heat equation with initial data $\phi$ and Dirichlet boundary conditions, as in (12), and we consider the associated heat content function $H_{\phi}(t)=\int_{\Omega} \phi_{t}$. It was first observed by van den Berg and Gilkey in [1] that the heat content admits an asymptotic series, as $t \rightarrow 0$, of type:

$$
H_{\phi}(t) \sim \int_{\Omega} \phi-\sum_{k=1}^{\infty} \beta_{k}(\phi) t^{k / 2} .
$$

for a family of invariants $\beta_{k}(\phi) \in \mathbf{R}$. The authors then computed the coefficients $\beta_{k}(\phi)$ up to $k=4$ (see also related work for the inhomogeneous case in [2]). In [21] a recursive formula for the calculation of the whole asymptotic series (18) was given: let us explain the outcome. As in Section 1.4, we fix a tubular neighborhood $U$ of $\partial \Omega$ where the distance function $\rho$ to the boundary of $\Omega$ is smooth and write $\nu=\nabla \rho$, a smooth vector field on $U$ which restricts to the unit normal vector on $\partial \Omega$. Consider the operator $N$ acting on $f \in C^{\infty}(U)$ as follows:

$$
\begin{aligned}
N f & =2\langle\nabla f, \nabla \rho\rangle-f \Delta \rho \\
& =2 \frac{\partial f}{\partial \nu}-\eta f
\end{aligned}
$$

Now let

$$
\mathcal{A}=\mathcal{A}(N, \Delta)
$$

be the algebra of differential operators acting on $C^{\infty}(U)$ and generated by the operator $N$ (of degree one), and the Laplacian $\Delta$ (of degree two). Then, $\mathcal{A}$ comes with a natural grading given by the degree, and each element of $\mathcal{A}$ will be a (non-commutative) polynomial in $N$ and $\Delta$. The main result of [21] states that there is a sequence $\left\{D_{k}\right\}$ of differential operators in the algebra $\mathcal{A}$ such that the coefficient $\beta_{k}(\phi)$ is obtained by integrating the function $D_{k} \phi$ over the boundary. The sequence $\left\{D_{k}\right\}$ is recursively defined, as follows. 
We start by defining operators $R_{k j}, S_{k j} \in \mathcal{A}(N, \Delta)$, depending on two non-negative integers $k, j$, by the following recursive rule:

$$
\left\{\begin{array}{l}
R_{k j}=-\left(N^{2}+\Delta\right) R_{k-1, j}+N S_{k-1, j} \\
S_{k j}=\Delta N R_{k-1, j}-\Delta S_{k-1, j}+N R_{k-1, j-1} \\
R_{00}=I, S_{00}=0, R_{k j}=S_{k j}=0 \quad \text { if } k<0 \text { or } j<0 .
\end{array}\right.
$$

Now set: $\{a, b\} \doteq \frac{\Gamma\left(a+b+\frac{1}{2}\right)}{(a+b) ! \Gamma\left(a+\frac{1}{2}\right)}$, and define the operators $Z_{n+1}, \alpha_{n} \in \mathcal{A}(N, \Delta)$ by:

$$
Z_{n+1}=\sum_{j=0}^{n}\{n+1, j-1\} R_{n+j, j}, \quad \alpha_{n}=\sum_{j=0}^{n+1}\{n, j\} S_{n+j, j}
$$

This is Theorem 2.1 in [21].

Theorem 11. Let $\beta_{k}(\phi)$ be the coefficient of $t^{k / 2}$ in the asymptotic expansion of the heat content (18), and let $D_{k} \in \mathcal{A}(N, \Delta)$ be the homogeneous polynomial of degree $k-1$ defined inductively by the formulas:

$$
\left\{\begin{array}{l}
D_{1}=\frac{2}{\sqrt{\pi}} I \\
D_{2 n}=\frac{1}{\sqrt{\pi}} \sum_{i=1}^{n} \frac{\Gamma\left(i+\frac{1}{2}\right) \Gamma\left(n-i+\frac{1}{2}\right)}{n !} D_{2 i-1} \alpha_{n-i} \\
D_{2 n+1}=\frac{1}{\sqrt{\pi}} Z_{n+1}+\frac{1}{\sqrt{\pi}} \sum_{i=1}^{n} \frac{i ! \Gamma\left(n-i+\frac{1}{2}\right)}{\Gamma\left(n+\frac{3}{2}\right)} D_{2 i} \alpha_{n-i}
\end{array}\right.
$$

Then, for all $k \geq 1$, we have $\beta_{k}(\phi)=\int_{\partial \Omega} D_{k} \phi$.

The sequence $\left\{D_{k}\right\}$ will be called the sequence of heat content operators of $\Omega$. We give below the explicit expression of the operators $D_{1}, \ldots, D_{6}$ (taken from Table 2.2 in [21]).

$$
\left\{\begin{array}{l}
D_{1}=\frac{2}{\sqrt{\pi}} I d, \quad D_{2}=\frac{1}{2} N, \quad D_{3}=\frac{1}{6 \sqrt{\pi}}\left(N^{2}-4 \Delta\right), \quad D_{4}=-\frac{1}{16}(\Delta N+3 N \Delta) \\
D_{5}=-\frac{1}{240 \sqrt{\pi}}\left(N^{4}+16 N^{2} \Delta+8 N \Delta N-48 \Delta^{2}\right) \\
D_{6}=\frac{1}{768}\left(\Delta N^{3}-N^{3} \Delta+N \Delta N^{2}-N^{2} \Delta N+40 N \Delta^{2}+8 \Delta^{2} N+16 \Delta N \Delta\right)
\end{array}\right.
$$

These results were refined in the paper 22 to obtain an asymptotic expansion of the heat flow valid at each point of the boundary (see Theorem 2.1 in [22]). 
Theorem 12. Let $\phi_{t}$ be the solution of the heat equation with initial data $\phi$ and Dirichlet boundary conditions, as in (12). Then, for all $y \in \partial \Omega$, there is an asymptotic series:

$$
\frac{\partial \phi}{\partial \nu}(t, y) \sim \frac{\phi(y)}{\sqrt{\pi}} \cdot \frac{1}{\sqrt{t}}+\sum_{k=0}^{\infty} \tilde{D}_{k} \phi(y) \cdot t^{k / 2} \quad \text { as } \quad t \rightarrow 0,
$$

where $\tilde{D}_{k} \in \mathcal{A}(N, \Delta)$ is the operator $\tilde{D}_{k}=\left(1+\frac{k}{2}\right) D_{k+2}$.

Taking unit initial data $\phi=1$ we see that the corresponding solution will be $u_{t}$, as in (11). Then, at each point $y \in \partial \Omega$, Theorem 12 will give the asymptotic expansion of the heat flow:

$$
\frac{\partial u}{\partial \nu}(t, y) \sim \frac{1}{\sqrt{\pi}} \cdot \frac{1}{\sqrt{t}}+\sum_{k=0}^{\infty} \tilde{D}_{k} 1(y) \cdot t^{k / 2} .
$$

If the heat flow is constant on $\partial \Omega$ for all times $t>0$, then necessarily the function $\tilde{D}_{k} 1$ (hence also the function $D_{k} 1$ ), when restricted to the boundary, will be constant for all $k$. We summarize these considerations in the following

Theorem 13. Assume that $\Omega$ has the constant flow property, and let $\left\{D_{k}\right\}$ be the sequence of heat content operators, as defined in (22). Then the function

$$
\left.B_{k} \doteq D_{k} 1\right|_{\partial \Omega}
$$

is constant on $\partial \Omega$ for all $k$.

\subsection{Proof of Theorem 7 for $k \leq 2$}

We finish this section by showing that the constant heat flow assumption and the expression of $D_{1}, D_{2}, D_{3}, D_{4}$ as given in (23) will imply that $\eta, \frac{\partial \eta}{\partial \nu}$ and $\frac{\partial^{2} \eta}{\partial \nu^{2}}$ are constant on $\partial \Omega$. This will give a hint for the general proof of Theorem 7 (which states that the normal derivative, of arbitrary order, of the function $\eta$ is constant on $\partial \Omega$ ).

Notice that as $N 1=-\eta$ and $\Delta 1=0$ we have, from table (23):

$$
B_{2}=-\frac{1}{2} \eta, \quad B_{3}=-\frac{1}{6 \sqrt{\pi}} N \eta, \quad B_{4}=\frac{1}{16} \Delta \eta .
$$

We now observe a useful splitting of the Laplace operator in the neighborhood $U$. Given $f \in C^{\infty}(U)$, we have

$$
\Delta f=-\frac{\partial^{2} f}{\partial \nu^{2}}+\eta \frac{\partial f}{\partial \nu}+\Delta_{T} f
$$

where $\Delta_{T} f$, the tangential Laplacian of $f$, is defined as follows. For $x \in U$, let $\rho^{-1}(r)$ be the level set of $\rho$ containing $x$ (so that $\rho(x)=r$ ). Then $\Delta_{T} f(x)$ is the Laplacian (for the induced metric on $\rho^{-1}(r)$ ) of the restriction of $f$ to $\rho^{-1}(r)$. We will call

$$
\Delta_{R} f \doteq-\frac{\partial^{2} f}{\partial \nu^{2}}+\eta \frac{\partial f}{\partial \nu}
$$


the radial Laplacian of $f$, so that, on $U: \Delta f=\Delta_{R} f+\Delta_{T} f$. The splitting (26) is easily proved by working with orthonormal frames of type $\left(e_{1}, \ldots, e_{n-1}, \nu\right)$, so that $\left(e_{1}, \ldots, e_{n-1}\right)$ will be an orthonormal frame of the equidistant through the point. Then, from formulae (25) and the splitting (26) we obtain:

$$
\left\{\begin{array}{l}
B_{2}=-\frac{1}{2} \eta \\
B_{3}=-\frac{1}{6 \sqrt{\pi}}\left(2 \frac{\partial \eta}{\partial \nu}-\eta^{2}\right) \\
B_{4}=\frac{1}{16}\left(-\frac{\partial^{2} \eta}{\partial \nu^{2}}+\eta \frac{\partial \eta}{\partial \nu}+\Delta_{T} \eta\right)
\end{array}\right.
$$

If $\Omega$ has the constant flow property, then Theorem 13 asserts that the functions $B_{k}$ are all constant on $\partial \Omega$ : an obvious inductive argument will then show that the normal derivatives $\eta, \frac{\partial \eta}{\partial \nu}$ and $\frac{\partial^{2} \eta}{\partial \nu^{2}}$ are also constant on $\partial \Omega$, which is Theorem 7 for $k \leq 2$.

Finally, we observe the following immediate proof of Theorem 2 when $\Omega$ is a domain in Euclidean or hyperbolic space. In fact, from table (28), we see that $\partial \Omega$ has constant mean curvature, hence, from a well-known result by Alexandrov, $\Omega$ must be a ball.

\section{Proof of Theorem 7}

In this section we write the invariants $B_{k}=\left.D_{k} 1\right|_{\partial \Omega}$ of Theorem 13 in terms of the normal derivatives of the function $\eta$ (as we have done it in Section 3.1 for $k \leq 4$ ) : this will be used to give an inductive proof of Theorem 7 .

We start by writing the invariants $B_{k}$ in a more suitable way. Given an operator $A$ of degree at least one in the algebra $\mathcal{A}(N, \Delta)$ defined in (19), we can decompose it as follows:

$$
A=\bar{A} N+\tilde{A} \Delta \text {, }
$$

for uniquely defined operators $\bar{A}$ (with $\operatorname{deg} \bar{A}=\operatorname{deg} A-1)$ and $\tilde{A}$ (with $\operatorname{deg} \tilde{A}=\operatorname{deg} A-2$ ). Clearly the map $A \rightarrow \bar{A}$ is linear, and for $A, B \in \mathcal{A}(N, \Delta)$ one has:

$$
\overline{A B}=A \bar{B} \text {. }
$$

For example, from table (23) we see:

$$
\bar{D}_{2}=\frac{1}{2} I, \quad \bar{D}_{3}=\frac{1}{6 \sqrt{\pi}} N, \quad \bar{D}_{4}=-\frac{1}{16} \Delta, \quad \bar{D}_{5}=-\frac{1}{240 \sqrt{\pi}}\left(N^{3}+8 N \Delta\right) .
$$

As $N 1=-\eta$ and $\Delta 1=0$ we see that $D_{k} 1=-\bar{D}_{k} \eta$. Then, Theorem 13 becomes the following statement.

Proposition 14. Assume that $\Omega$ has the constant flow property. Then the function

$$
B_{k}=-\left.\bar{D}_{k} \eta\right|_{\partial \Omega}
$$

is constant on $\partial \Omega$ for all $k$. 
Our next task is to determine the coefficient of the normal derivative of highest order in a given homogeneous operator belonging to the algebra $\mathcal{A}(N, \Delta)$. Define a function, called the weight:

$$
w: \mathcal{A}(N, \Delta) \rightarrow \mathbf{R}
$$

by setting $w(I)=1, w(N)=2, w(\Delta)=-1$ and then extending $w$ to $\mathcal{A}$ as an algebra homomorphism. For example, from list (31):

$$
w\left(\bar{D}_{2}\right)=\frac{1}{2}, \quad w\left(\bar{D}_{3}\right)=\frac{1}{3 \sqrt{\pi}}, \quad w\left(\bar{D}_{4}\right)=\frac{1}{16}, \quad w\left(\bar{D}_{5}\right)=\frac{1}{30 \sqrt{\pi}} .
$$

From the decomposition $\Delta=\Delta_{R}+\Delta_{T}$ of (26) one sees why $\Delta$ should have weight -1 . The inductive step is based on the following fact.

Proposition 15. Fix an integer $k \geq 0$ and assume that the function $\eta$, together with all of its normal derivatives up and including the order $k$, restricts to a constant function on $\partial \Omega$. Then:

(a) If $A$ is an operator in $\mathcal{A}(N, \Delta)$ homogeneous of degree $k+1$, then:

$$
\left.A \eta\right|_{\partial \Omega}=\left.w(A) \frac{\partial^{k+1} \eta}{\partial \nu^{k+1}}\right|_{\partial \Omega}+c_{k}
$$

where $w(A)$ is the weight of $A$ and $c_{k}$ is constant on $\partial \Omega$.

(b) If $B_{k+3} \in C^{\infty}(\partial \Omega)$ is the invariant of Proposition 14, then, for all $y \in \partial \Omega$ :

$$
B_{k+3}(y)=-w\left(\bar{D}_{k+3}\right) \frac{\partial^{k+1} \eta}{\partial \nu^{k+1}}(y)+b_{k}
$$

where $b_{k}$ is a constant which does not depend on $y \in \partial \Omega$.

We will give the proof of this proposition in Section 4.3 below. The following result is the main combinatorial fact needed in the proof of Theorem 7 , its proof will take the rest of the paper, starting from the next section.

Theorem 16. Let $\left\{D_{k}\right\}$ be the sequence of heat content operators, and consider the operators $\bar{D}_{k}$ defined in (29). Then, one has for all $n \geq 1$ :

$$
\left\{\begin{array}{l}
w\left(\bar{D}_{2 n}\right)=\frac{2}{4^{n} n !} \\
w\left(\bar{D}_{2 n+1}\right)=\frac{1}{\sqrt{\pi}} \cdot \frac{1}{2^{n-1}(2 n+1) ! !}
\end{array}\right.
$$

In particular, $w\left(\bar{D}_{k}\right) \neq 0$ for all $k$.

We observe the following easy consequence of Proposition 15 and Theorem 16. 
Proposition 17. Assume that $\Omega$ is a domain such that the invariants $B_{2}, \ldots, B_{k}$ are constant on $\partial \Omega$ for some $k \geq 2$. Then the functions $\eta, \frac{\partial \eta}{\partial \nu}, \ldots, \frac{\partial^{k-2} \eta}{\partial \nu^{k-2}}$ are constant on $\partial \Omega$.

Proof. The proof is by induction on $k$. The statement is true for $k=2$, because $B_{2}=-\frac{1}{2} \eta$ (see table (25) ). Assume that it is true for the integer $k$, and assume that $B_{2}, \ldots, B_{k+1}$ are constant. We have to show that $\frac{\partial^{k-1} \eta}{\partial \nu^{k-1}}$ is constant. Now, we know that $\eta, \frac{\partial \eta}{\partial \nu}, \ldots, \frac{\partial^{k-2} \eta}{\partial \nu^{k-2}}$ are all constant by the inductive hypothesis; by Proposition 15b we have:

$$
B_{k+1}=a_{k} \frac{\partial^{k-1} \eta}{\partial \nu^{k-1}}+c_{k}
$$

where $a_{k}$ is the weight of $-\bar{D}_{k+1}$, which is non-zero by Theorem [16, and $c_{k}$ is constant. This immediately shows that $\frac{\partial^{k-1} \eta}{\partial \nu^{k-1}}$ must also be constant and the assertion follows.

We can now prove Theorem 7 and Theorem 4 .

\subsection{Proof of Theorem 7}

Assume that $\Omega$ has the constant flow property. Recall that we need to show that the $k$-th normal derivative of the function $\eta$ is constant on $\partial \Omega$ for all $k \geq 0$. By Proposition 14 the function $B_{k}$ is constant on $\partial \Omega$ for all $k$. Then, the conclusion follows immediately from Proposition 17.

\subsection{Proof of Theorem 4}

We consider the heat content $\tilde{H}_{f}(t)$ with boundary data $f \in C_{0}^{\infty}(\partial \Omega)$, as defined in (44). From (16) we know that

$$
\tilde{H}_{f}^{\prime}(t)=\int_{\partial \Omega} f \frac{\partial u_{t}}{\partial \nu}
$$

Substituting for $\partial u_{t} / \partial \nu$ the expansion in (24) , integrating the asymptotic series term to term, and recalling that $\tilde{D}_{k} 1=\left(1+\frac{k}{2}\right) D_{k+2} 1=\left(1+\frac{k}{2}\right) B_{k+2}$ we see that, as $t \rightarrow 0$ :

$$
\tilde{H}_{f}(t) \sim \sum_{k=2}^{\infty} \int_{\partial \Omega} f B_{k} \cdot t^{\frac{k}{2}}
$$

(the series starts with $k=2$ because $f$ integrates to zero on $\partial \Omega$ ).

Proof of (a). By assumption, $\tilde{H}_{f}(t)=o(t)$ as $t \rightarrow 0$ hence, from (32): $\int_{\partial \Omega} f B_{2}=0$. This happens for all $f \in C_{0}^{\infty}(\partial \Omega)$ hence $B_{2}$ must be constant. From table (25) we see that $\eta=-2 B_{2}$ must be constant, hence, as $\partial \Omega$ is compact and has constant mean curvature, $\Omega$ must be a ball by the Alexandrov theorem, which is valid in $\mathbf{R}^{n}$ and $\mathbf{H}^{n}$. 
Proof of (b). We have by assumption that $\Omega \subseteq \mathbf{S}^{n}$ and that $\tilde{H}_{f}(t) \sim o\left(t^{k / 2}\right)$ for some $k \geq 2$, and for all $f \in C_{0}^{\infty}(\partial \Omega)$. We must then prove that all mean curvatures of order $\leq k-1$ are constant on $\partial \Omega$.

Step 1. The invariants $B_{2}, \ldots, B_{k}$ are constant on $\partial \Omega$.

This follows immediately because, from (32) and our assumption, $\int_{\partial \Omega} f B_{j}=0$ for all $j \leq k$.

Step 2. The functions $\eta, \frac{\partial \eta}{\partial \nu}, \ldots, \frac{\partial^{k-2} \eta}{\partial \nu^{k-2}}$ are constant on $\partial \Omega$.

This follows from Step 1 and Proposition 17.

Define a field of endomorphisms $S$ of the tangent space of the tubular neighborhood $U$ by:

$$
S(X)=-\nabla_{X} \nu
$$

where $\nu=\nabla \rho$ is everywhere normal to the equidistants. Note that $S(\nu)=-\nabla_{\nu} \nu$ is identically zero because $\nu$ is of unit length and tangent to the normal geodesics. When restricted to the tangent space of $\rho^{-1}(r)$ (in particular, the tangent space of $\partial \Omega$ ) the endomorphism $S$ is just the shape operator, hence, on $U$, one has $\eta=\operatorname{tr} S$. A straightforward calculation shows that

$$
\nabla_{\nu} S=S^{2}+R_{\nu}
$$

where $R_{\nu}(X)=R(\nu, X) \nu$ and $R$ is the Riemann tensor of the ambient manifold $\Omega$.

Step 3. The traces $\operatorname{tr}(S), \operatorname{tr}\left(S^{2}\right), \ldots, \operatorname{tr}\left(S^{k-1}\right)$ are constant on $\partial \Omega$.

Since $B_{2}=-\frac{1}{2} \eta$ is constant on $\partial \Omega$, also $\operatorname{tr}(S)=\eta$ is constant on $\partial \Omega$. Now $\nabla_{\nu}$ commutes with taking traces, and one has $\operatorname{tr}\left(R_{\nu}\right)=\operatorname{Ric}(\nu, \nu)$, where Ric denotes the Ricci tensor. If $\Omega \subseteq \mathbf{S}^{n}$, taking traces in (33) one then gets:

$$
\frac{\partial \eta}{\partial \nu}=\operatorname{tr}\left(S^{2}\right)+n-1
$$

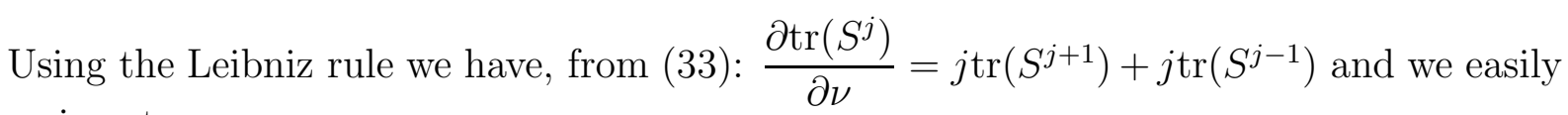
arrive at:

$$
\frac{\partial^{m} \eta}{\partial \nu^{m}}=m ! \operatorname{tr}\left(S^{m+1}\right)+\sum_{j=0}^{m-1} a_{j} \operatorname{tr}\left(S^{j}\right)
$$

where each $a_{j}$ is a constant. Induction on $k$ shows that Step 2 implies Step 3.

Step 4. The mean curvatures $E_{1}, \ldots, E_{k-1}$ are constant on $\partial \Omega$.

This fact is a consequence of Step 3 and the well-known Newton's identities, which relate the $r$-th elementary symmetric function of the eigenvalues $k_{1}, \ldots, k_{n-1}$ with the sums of 
the powers of the eigenvalues, that is, the trace of the powers of $S: \operatorname{tr}\left(S^{m}\right)=\sum_{j=1}^{n-1} k_{j}^{m}$. Precisely, one has, for all $k$ :

$$
k E_{k}=\sum_{i=1}^{k}(-1)^{i-1} E_{k-i} \operatorname{tr}\left(S^{i}\right) .
$$

Hence we have a scheme like this:

$$
\begin{aligned}
& E_{1}=\operatorname{tr}(S) \\
& E_{2}=E_{1} \operatorname{tr}(S)-\operatorname{tr}\left(S^{2}\right) \\
& E_{3}=E_{2} \operatorname{tr}(S)-E_{1} \operatorname{tr}\left(S^{2}\right)+\operatorname{tr}\left(S^{3}\right)
\end{aligned}
$$

showing that, if $\operatorname{tr}\left(S^{i}\right)$ is constant for all $i \leq k-1$, then also $E_{1}, \ldots, E_{k-1}$ are constant. Proof of (c). It simply follows from the fact that, if $\tilde{H}_{f}(t)=o\left(t^{\frac{n}{2}}\right)$ for all $f \in C_{0}^{\infty}(\partial \Omega)$, then taking $k=n$ in the previous statement (b) we see that all mean curvatures $E_{1}, \ldots, E_{n-1}$ are constant, hence the characteristic polynomial of $S$ is constant on $\partial \Omega$, which implies that $\partial \Omega$ is isoparametric by Theorem [6. The proof is now complete.

\subsection{Proof of Proposition 15}

The proposition could be proved by writing the operator $A$ in local normal coordinates $(r, y)$ around a boundary point $y \in \partial \Omega$. However, we give here a direct argument. Before giving the proof, we introduce the following terminology.

- We say that $\phi \in C^{\infty}(U)$ has level $k$ if $k$ is the largest integer (including possibly $k=+\infty)$ such that $\phi, \frac{\partial \phi}{\partial \nu}, \ldots, \frac{\partial^{k} \phi}{\partial \nu^{k}}$ restrict to constant functions on $\partial \Omega$.

By convention, if $\left.\phi\right|_{\partial \Omega}$ is non constant then we say that $\phi$ has level $-\infty$. Note that if $\left.\phi\right|_{\partial \Omega}$ is constant then $\phi$ has level $k \geq 0$, while radial functions on $U$ have level $+\infty$. In fact, if $\phi=f \circ \rho$ for a smooth function $f:[0, \epsilon) \rightarrow \mathbf{R}$, then $\frac{\partial^{k} \phi}{\partial \nu^{k}}=f^{(k)} \circ \rho$ which, restricted to $\partial \Omega$, takes the constant value $f^{(k)}(0)$.

By using Taylor expansion in the normal direction based at a generic point of the boundary, we easily obtain the following characterization.

Lemma 18. A function $\phi \in C^{\infty}(U)$ has level at least $k$ if and only if there exist smooth functions $f:[0, \epsilon) \rightarrow \mathbf{R}$ and $\psi \in C^{\infty}(U)$ such that, on $U:$

$$
\phi=f \circ \rho+\rho^{k+1} \psi .
$$

In that case $f(r)$ will be a polynomial of degree $k$.

We will also need the following fact. 
Lemma 19. Assume that $\eta$ has level at least $k$. If $\phi \in C^{\infty}(U)$ has level $h$, with $h \leq k$, then:

a) $N \phi$ has level at least $h-1$,

b) $\Delta_{R} \phi$ has level at least $h-2$,

c) $\Delta_{T} \phi$ has level at least $h$.

In particular, if $T$ is a polynomial of degree $m \leq h$ in the operators $N, \Delta_{R}, \Delta_{T}$ then $T \phi$ has level at least $h-m$.

Proof. The assumptions imply existence of smooth functions $f, g$ on $[0, \epsilon)$ and $\psi, \xi$ on $U$ such that

$$
\left\{\begin{array}{l}
\phi=f \circ \rho+\rho^{h+1} \psi \\
\eta=g \circ \rho+\rho^{k+1} \xi
\end{array}\right.
$$

One has:

$$
\frac{\partial \phi}{\partial \nu}=f^{\prime} \circ \rho+\rho^{h}\left((h+1) \psi+\rho \frac{\partial \psi}{\partial \nu}\right)
$$

and by the previous lemma $\frac{\partial \phi}{\partial \nu}$ has level at least $h-1$. Since $h \leq k$ one sees immediately that $\eta \phi$ has level at least $h$, hence $N \phi$ has level at least $h-1$, showing 1). Part 2) is proved similarly; as for part 3) just observe that, as radial functions are constant on the level surfaces of $\rho$, one has $\Delta_{T} \phi=\rho^{h+1} \Delta_{T} \psi$ showing that $\Delta_{T} \phi$ has level at least $h$.

The last assertion is now clear, as an obvious induction shows.

We now can prove Proposition [15, part (a). Given an operator $A \in \mathcal{A}(N, \Delta)$ of homogeneous degree $k+1$, recall the splitting $\Delta=\Delta_{R}+\Delta_{T}$. Accordingly, we can split

$$
A=A_{R}+A_{T},
$$

where $A_{R}$ is a polynomial in $N$ and $\Delta_{R}$ and $A_{T}$ contains at least one $\Delta_{T}$. For example, if $A=N \Delta^{2}$ then $A_{R}=N \Delta_{R}^{2}$ and $A_{T}=N \Delta_{R} \Delta_{T}+N \Delta_{T} \Delta_{R}+N \Delta_{T}^{2}$.

Now $A_{R}$ derives functions only radially, and has degree $k+1$ : then, the highest order of the normal derivative occurring in $A \eta$ is $k+1$. By definition of weight one sees easily that, on $U$, one has:

$$
A_{R} \eta=w(A) \frac{\partial^{k+1} \eta}{\partial \nu^{k+1}}+P\left(\eta, \frac{\partial \eta}{\partial \nu}, \ldots, \frac{\partial^{k} \eta}{\partial \nu^{k}}\right)
$$

where the second term in the right is a polynomial in the functions $\eta, \frac{\partial \eta}{\partial \nu}, \ldots, \frac{\partial^{k} \eta}{\partial \nu^{k}}$. By our assumption, all these functions restrict to constants on $\partial \Omega$. By taking the restriction to the boundary we see

$$
\left.A_{R} \eta\right|_{\partial \Omega}=\left.w(A) \frac{\partial^{k+1} \eta}{\partial \nu^{k+1}}\right|_{\partial \Omega}+c_{k}
$$


where $c_{k}$ is constant. As $A \eta=A_{R} \eta+A_{T} \eta$, the final assertion now follows by proving that

$$
\left.A_{T} \eta\right|_{\partial \Omega}=0
$$

In order to do that, first observe that $A_{T}$ is a sum of terms of type $B \Delta_{T} C$, where $B$ is a polynomial in $N$ and $\Delta_{R}$, and $C$ is a polynomial in $N, \Delta_{R}, \Delta_{T}$. If $B$ has degree $h$ (possibly $h=0$ ) then $C$ has degree $k-h-1$. By assumption $\eta$ has level at least $k$, hence (by Lemma 19) $C \eta$ has level at least $k-(k-h-1)=h+1$ and, by Lemma 18, there exist smooth functions $f$ on $[0, \epsilon)$ and $\psi$ on $U$ such that:

$$
C \eta=f \circ \rho+\rho^{h+2} \psi .
$$

Therefore $\Delta_{T} C \eta=\rho^{h+2} \Delta_{T} \psi$ vanishes on the boundary together with all its normal derivatives up and including the order $h+1$. As $B$ has order $h$ one must have $B\left(\rho^{h+2} \Delta_{T} \psi\right)=0$ on the boundary, hence

$$
\left.B \Delta_{T} C \eta\right|_{\partial \Omega}=0
$$

as asserted. This proves part (a).

For part (b), recall that $B_{k+3}=-\bar{D}_{k+3} \eta$. Hence it is enough to apply (a) to the operator $A=\bar{D}_{k+3}$, which is homogeneous of degree $k+1$.

\section{Theorem 16: the two main steps and the proof}

At this point all results of the paper are proved, except Theorem 16. The proof of Theorem 16 is combinatorial, and will take the rest of the paper. We have split the proof in several sections.

First, recall the heat content operators $\left\{D_{k}\right\}$ as defined in Theorem 11, and recall the barred operators $\left\{\bar{D}_{k}\right\}$ defined by the rule (29) $: D_{k}=\bar{D}_{k} N+\tilde{D}_{k} \Delta$. If $w\left(\bar{D}_{k}\right)$ denotes the weight of $\bar{D}_{k}$, we need to prove the identities:

$$
\left\{\begin{array}{l}
w\left(\bar{D}_{2 n}\right)=\frac{2}{4^{n} n !}, \\
w\left(\bar{D}_{2 n+1}\right)=\frac{1}{\sqrt{\pi}} \cdot \frac{1}{2^{n-1}(2 n+1) ! !}
\end{array}\right.
$$

for all integers $n$. There are two main steps: in Proposition 20 we compute the weight of $D_{k}$, and in Proposition 21 we compute the weight of the barred operators $\bar{\alpha}_{n}, \bar{Z}_{n+1}$ defined in (21). Assuming Propositions 20 and 21 we prove Theorem 16 later in this section. The proof of Proposition 20 will then be given in Section 6, while the proof of Proposition 21 is more involved, and will be given in the last sections of the paper (Sections 7,8 and the Appendix).

As a first step we compute the weights of $\left\{D_{k}\right\}$, by a functorial argument. 
Proposition 20. One has, for all $n \geq 1$ :

$$
\left\{\begin{array}{l}
w\left(D_{2 n}\right)=\frac{1}{n !} \\
w\left(D_{2 n+1}\right)=\frac{1}{\Gamma\left(n+\frac{3}{2}\right)}=\frac{1}{\sqrt{\pi}} \cdot \frac{2^{n+1}}{(2 n+1) ! !}
\end{array}\right.
$$

Proof: Section 6.

Then, we compute the weights of the barred operators $\bar{\alpha}_{n}$ and $\bar{Z}_{n+1}$ (this is perhaps the main combinatorial difficulty).

Proposition 21. One has:

$$
\left\{\begin{array}{l}
w\left(\bar{\alpha}_{0}\right)=\frac{1}{2} \\
w\left(\bar{\alpha}_{n}\right)=-\frac{3}{2^{n+1}(2 n-1) ! !} \quad \text { for all } n \geq 1 \\
w\left(\bar{Z}_{n+1}\right)=-\frac{1}{2^{n-1}(2 n+1) ! !} \quad \text { for all } n \geq 1
\end{array}\right.
$$

Proof: Sections 7, 8 and the Appendix.

\subsection{Proof of Theorem 16}

Taking the bar on both sides of the recurrence scheme (22) we see, thanks to rule $\overline{A B}=$ $A \bar{B}$, that $\bar{D}_{1}=0$ and, for all $n \geq 1$ :

$$
\left\{\begin{array}{l}
\bar{D}_{2 n}=\frac{1}{\sqrt{\pi}} \sum_{i=1}^{n} \frac{\Gamma\left(i+\frac{1}{2}\right) \Gamma\left(n-i+\frac{1}{2}\right)}{n !} D_{2 i-1} \bar{\alpha}_{n-i} \\
\bar{D}_{2 n+1}=\frac{1}{\sqrt{\pi}} \bar{Z}_{n+1}+\frac{1}{\sqrt{\pi}} \sum_{i=1}^{n} \frac{i ! \Gamma\left(n-i+\frac{1}{2}\right)}{\Gamma\left(n+\frac{3}{2}\right)} D_{2 i} \bar{\alpha}_{n-i}
\end{array}\right.
$$

We first prove that

$$
w\left(\bar{D}_{2 n}\right)=\frac{2}{4^{n} n !}
$$

First, recall the recurrence law for the Gamma function:

$$
\Gamma\left(\frac{1}{2}\right)=\sqrt{\pi}, \quad \Gamma(1+x)=x \Gamma(x), \quad \text { so that } \quad \Gamma\left(n+\frac{1}{2}\right)=\sqrt{\pi} \cdot \frac{(2 n-1) ! !}{2^{n}} .
$$

From Proposition 21, $w\left(\bar{\alpha}_{n}\right)$ can be re-written:

$$
w\left(\bar{\alpha}_{n}\right)=-\frac{3 \sqrt{\pi}}{2^{2 n+1} \Gamma\left(n+\frac{1}{2}\right)} .
$$


By taking weights in the first relation of (35) we obtain:

$$
\begin{aligned}
\sqrt{\pi} n ! w\left(\bar{D}_{2 n}\right) & =\sum_{i=1}^{n} \Gamma\left(i+\frac{1}{2}\right) \Gamma\left(n-i+\frac{1}{2}\right) w\left(D_{2 i-1}\right) w\left(\bar{\alpha}_{n-i}\right) \\
& =\sum_{i=1}^{n-1} \Gamma\left(i+\frac{1}{2}\right) \Gamma\left(n-i+\frac{1}{2}\right) w\left(D_{2 i-1}\right) w\left(\bar{\alpha}_{n-i}\right)+\Gamma\left(n+\frac{1}{2}\right) \Gamma\left(\frac{1}{2}\right) w\left(D_{2 n-1}\right) w\left(\bar{\alpha}_{0}\right)
\end{aligned}
$$

By Proposition $\left[20\right.$ we get $w\left(D_{2 i-1}\right)=\frac{1}{\Gamma\left(i+\frac{1}{2}\right)}$ and from (37) we obtain for $i \leq n-1$ :

$$
w\left(\bar{\alpha}_{n-i}\right)=-\frac{3 \sqrt{\pi}}{2^{2(n-i)+1} \Gamma\left(n-i+\frac{1}{2}\right)}=-\frac{\sqrt{\pi} \cdot 3 \cdot 4^{i}}{2^{2 n+1} \Gamma\left(n-i+\frac{1}{2}\right)} .
$$

Hence from (38)

$$
\sqrt{\pi} n ! w\left(\bar{D}_{2 n}\right)=-\frac{3 \sqrt{\pi}}{2^{2 n+1}} \sum_{i=1}^{n-1} 4^{i}+\frac{\sqrt{\pi}}{2}
$$

As $\sum_{i=1}^{n-1} 4^{i}=\frac{4^{n}-4}{3}$ we get $w\left(\bar{D}_{2 n}\right)=\frac{2}{4^{n} n !}$ which is the first identity in Theorem 16, The second assertion is proved similarly, by taking weights in the second relation of (35). We omit the details, which are straightforward.

\section{Theorem 16, step 1: proof of Proposition 20}

We start from the following lemma.

Lemma 22. Let $\Omega$ be any domain, let $\phi \in C^{\infty}(\Omega)$ and assume that $\phi=0$ on $\partial \Omega$. Let $\left\{D_{k}\right\}$ be the sequence of heat content operators. Then, for all $j \geq 1$ :

$$
\int_{\partial \Omega} D_{j+2} \phi=-\frac{2}{j+2} \int_{\partial \Omega} D_{j} \Delta \phi
$$

Proof. Recall that $\phi_{t}$ is the solution of the heat equation on $\Omega$ with initial data $\phi$ and Dirichlet boundary conditions. One knows from Section 3 that, as $t \rightarrow 0$, the heat content has an asymptotic series

$$
\int_{\Omega} \phi_{t} \sim \int_{\Omega} \phi-\sum_{k=1}^{\infty} \beta_{k}(\phi) t^{\frac{k}{2}}
$$

Differentiating both sides with respect to $t$ we obtain:

$$
\frac{d}{d t} \int_{\Omega} \phi_{t} \sim-\sum_{k=1}^{\infty} \frac{k}{2} \beta_{k}(\phi) t^{\frac{k-2}{2}} \sim-\sum_{j=-1}^{\infty} \frac{j+2}{2} \beta_{j+2}(\phi) t^{\frac{j}{2}}
$$


On the other hand we also know that $\int_{\Omega} \phi_{t}=\int_{\Omega} \phi u_{t}$ (see (14)). Therefore, as $\phi$ and $u_{t}$ vanish on the boundary:

$$
\begin{aligned}
\frac{d}{d t} \int_{\Omega} \phi_{t} & =\frac{d}{d t} \int_{\Omega} \phi u_{t} \\
& =-\int_{\Omega} \phi \Delta u_{t} \\
& =-\int_{\Omega} \Delta \phi \cdot u_{t}+\int_{\partial \Omega} u_{t} \frac{\partial \phi}{\partial \nu}-\int_{\partial \Omega} \frac{\partial u_{t}}{\partial \nu} \phi \\
& =-\int_{\Omega} \Delta \phi \cdot u_{t} \\
& =-\int_{\Omega}(\Delta \phi)_{t} \\
& \sim-\int_{\Omega} \Delta \phi+\sum_{j=1}^{\infty} \beta_{j}(\Delta \phi) t^{\frac{j}{2}}
\end{aligned}
$$

We now equate the asymptotic series in (40) and (41): as $\phi$ vanishes on the boundary, one easily verifies that $\beta_{1}(\phi)=0, \beta_{2}(\phi)=\int_{\partial \Omega} \partial \phi / \partial \nu=\int_{\Omega} \Delta \phi$ and, for $j \geq 1$ :

$$
-\frac{j+2}{2} \beta_{j+2}(\phi)=\beta_{j}(\Delta \phi) \text {. }
$$

Recalling from Theorem 11 that $\beta_{j}(\phi)=\int_{\partial \Omega} D_{j} \phi$ we get the assertion.

We can now prove the proposition. The crucial observation is that the coefficients of $D_{k}$ are universal, and do not depend on the domain $\Omega$ : to compute the weight of $D_{k}$ we can just work on $\Omega=[0,1]$. Note that then $\partial \Omega=\{0,1\}$, and that the radial vector field $\nu$, restricted to the neighborhood $U=\Omega \backslash\left\{\frac{1}{2}\right\}$ of $\partial \Omega$ where $\rho$ is regular, is given by:

$$
\nu= \begin{cases}\frac{d}{d x} & \text { on } \quad\left[0, \frac{1}{2}\right) \\ -\frac{d}{d x} & \text { on }\left(\frac{1}{2}, 1\right] .\end{cases}
$$

As the distance function $\rho$ is linear on $U$, we have $\eta=\Delta \rho=0$, hence

$$
N \phi=2 \frac{\partial \phi}{\partial \nu}, \quad \Delta \phi=-\frac{\partial^{2} \phi}{\partial \nu^{2}}
$$

and one immediately sees that, if $A \in \mathcal{A}(N, \Delta)$ is homogeneous of degree $k$, then:

$$
A \phi=w(A) \frac{\partial^{k} \phi}{\partial \nu^{k}} .
$$


Now, as $D_{j+2}$ is homogeneous of degree $j+1$ :

$$
\int_{\partial \Omega} D_{j+2} \phi=w\left(D_{j+2}\right) \int_{\partial \Omega} \frac{\partial^{j+1} \phi}{\partial \nu^{j+1}} .
$$

On the other hand, assuming that $\phi=0$ on $\partial \Omega$, we have by the lemma:

$$
\begin{aligned}
\int_{\partial \Omega} D_{j+2} \phi & =-\frac{2}{j+2} \int_{\partial \Omega} D_{j} \Delta \phi \\
& =-\frac{2}{j+2} w\left(D_{j}\right) \int_{\partial \Omega} \frac{\partial^{j-1} \Delta \phi}{\partial \nu^{j-1}} \\
& =\frac{2}{j+2} w\left(D_{j}\right) \int_{\partial \Omega} \frac{\partial^{j+1} \phi}{\partial \nu^{j+1}}
\end{aligned}
$$

By (42) and (43) we conclude that, for all $\phi \in C^{\infty}(\Omega)$ which vanish on the boundary one has:

$$
\left(w\left(D_{j+2}\right)-\frac{2}{j+2} w\left(D_{j}\right)\right) \int_{\partial \Omega} \frac{\partial^{j+1} \phi}{\partial \nu^{j+1}}=0 .
$$

We can always choose $\phi$ so that the integral on the right does not vanish; hence, for all $j$ :

$$
w\left(D_{j+2}\right)=\frac{2}{j+2} w\left(D_{j}\right) .
$$

As $w\left(D_{2}\right)=1$ (see table (23) $)$ we obtain $w\left(D_{2 n}\right)=\frac{1}{n !}$ as asserted. Similarly, since $w\left(D_{1}\right)=\frac{2}{\sqrt{\pi}}$ we obtain $w\left(D_{2 n+1}\right)=\frac{1}{\Gamma\left(n+\frac{3}{2}\right)}$. The proof is complete.

\section{Theorem 16, step 2: proof of Proposition 21}

Perhaps, this is the step which is more involved from a combinatorial point of view. We first make a change of variables in the original definition of the operators $R_{n j}, S_{n j}$ in (20) to simplify the subsequent calculations. Then, the proof of Proposition 21 will reduce to the proof of Proposition 23 below, which in turn will be given in the last section.

For non-negative integers $n$ and $j$, let us introduce new operators $P_{n j}, Q_{n j} \in \mathcal{A}(N, \Delta)$ as follows:

$$
\left\{\begin{array}{l}
P_{n j}=-\frac{2}{4^{j}} R_{n+j, j}, \\
Q_{n j}=-\frac{4}{4^{j}} S_{n+j, j} .
\end{array}\right.
$$

Then, one easily shows that the defining scheme (20) takes the form:

$$
\left\{\begin{array}{l}
P_{n j}=-\left(N^{2}+\Delta\right) P_{n-1, j}+\frac{1}{2} N Q_{n-1, j} \\
Q_{n j}=2 \Delta N P_{n-1, j}-\Delta Q_{n-1, j}+\frac{1}{2} N P_{n, j-1} \\
P_{00}=-2 I, \quad Q_{00}=0
\end{array}\right.
$$


with the understanding that $P_{n j}$ and $Q_{n j}$ are zero whenever $n$ or $j$ is negative. Introduce the sequence

$$
a_{n}=\left(\begin{array}{c}
2 n-1 \\
n
\end{array}\right)=\frac{2^{n-1}(2 n-1) ! !}{n !} \text { for } n \geq 1 .
$$

The first few terms are $1,3,10,35,126,462,1716,6735, \ldots$ Recalling the relations (36) of the Gamma function it is straightforward to verify that the operators $Z_{n+1}$ and $\alpha_{n}$ of (21) can be written, for $n \geq 1$ :

$$
\left\{\begin{array}{l}
Z_{n+1}=-\frac{1}{2^{n-1}(2 n+1) ! !} \sum_{j=0}^{n} a_{n+j} P_{n j} \\
\alpha_{n}=-\frac{1}{2^{n+1}(2 n-1) ! !} \sum_{j=0}^{n+1} a_{n+j} Q_{n j}
\end{array}\right.
$$

For $n=0$, it can be directly checked from the original recursive scheme (21) that

$$
\alpha_{0}=\frac{1}{2} N
$$

(in fact $\left.\{0,1\}=\frac{1}{2}, S_{00}=0, S_{11}=N\right)$. Then $\bar{\alpha}_{0}=\frac{1}{2} I$ and $w\left(\bar{\alpha}_{0}\right)=\frac{1}{2}$.

Then, we need to verify the value of $w\left(\bar{\alpha}_{n}\right)$ and $w\left(\bar{Z}_{n+1}\right)$ given in Proposition 21 only when $n \geq 1$. By taking bars in (46) and then taking weights we see

$$
\left\{\begin{array}{l}
w\left(\bar{Z}_{n+1}\right)=-\frac{1}{2^{n-1}(2 n+1) ! !} \sum_{j=0}^{n} a_{n+j} w\left(\bar{P}_{n j}\right) \\
w\left(\bar{\alpha}_{n}\right)=-\frac{1}{2^{n+1}(2 n-1) ! !} \sum_{j=0}^{n+1} a_{n+j} w\left(\bar{Q}_{n j}\right) .
\end{array}\right.
$$

From the scheme (44) we see that

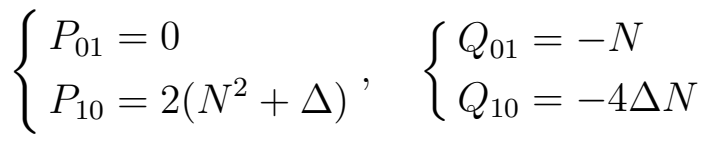

so that

$$
\left\{\begin{array}{l}
\bar{P}_{01}=0 \\
\bar{P}_{10}=2 N
\end{array}, \quad\left\{\begin{array}{l}
\bar{Q}_{01}=-I \\
\bar{Q}_{10}=-4 \Delta
\end{array} .\right.\right.
$$

Taking the bar in the scheme (44) we see that $\bar{P}_{n j}$ and $\bar{Q}_{n j}$ are uniquely determined by the scheme:

$$
\left\{\begin{array}{l}
\bar{P}_{n j}=-\left(N^{2}+\Delta\right) \bar{P}_{n-1, j}+\frac{1}{2} N \bar{Q}_{n-1, j} \\
\bar{Q}_{n j}=2 \Delta N \bar{P}_{n-1, j}-\Delta \bar{Q}_{n-1, j}+\frac{1}{2} N \bar{P}_{n, j-1} \\
\bar{P}_{01}=0, \quad \bar{P}_{10}=2 N, \quad \bar{Q}_{01}=-I, \quad \bar{Q}_{10}=-4 \Delta .
\end{array}\right.
$$


For non-negative integers $n$ and $j$ we now define real numbers (actually integers) by:

$$
T_{n j} \doteq w\left(\bar{P}_{n j}\right), \quad U_{n j} \doteq w\left(\bar{Q}_{n j}\right) .
$$

Recalling that taking weights is an algebra homomorphism, we see from (48) that $T_{n j}$ and $U_{n j}$ must satisfy the recurrence scheme:

$$
\left\{\begin{array}{l}
T_{n j}=-3 T_{n-1, j}+U_{n-1, j} \\
U_{n j}=-4 T_{n-1, j}+U_{n-1, j}+T_{n, j-1} \\
\left(\begin{array}{l}
T_{01} \\
U_{01}
\end{array}\right)=\left(\begin{array}{c}
0 \\
-1
\end{array}\right), \quad\left(\begin{array}{l}
T_{10} \\
U_{10}
\end{array}\right)=\left(\begin{array}{l}
4 \\
4
\end{array}\right)
\end{array}\right.
$$

It is now clear that Proposition 21 is implied by (47) and the following result.

Proposition 23. Let $T_{n j}$ and $U_{n j}$ be the integers defined by the scheme (49) and let $\left\{a_{n}\right\}$ be the sequence defined in (45). Then, for all $n \geq 1$ one has:

$$
\sum_{j=0}^{n} a_{n+j} T_{n j}=1, \quad \sum_{j=0}^{n+1} a_{n+j} U_{n j}=3 .
$$

\section{Last step: proof of Proposition 23}

The proof depends on a combinatorial result (see Lemma 24), which can be proved in the framework of orthogonal polynomials and Hankel transforms. For the proof, we refer to the Appendix (Section 91) where we will use the results in [26] (see also [8] for related work with the sequence of Catalan numbers).

For the sequence

$$
\left\{a_{n}\right\}=\left\{\left(\begin{array}{c}
2 n-1 \\
n
\end{array}\right)\right\}_{n \geq 1}=\{1,3,10,35,126,462,1716,6735, \ldots\}
$$

defined in (45) introduce the infinite matrices:

$$
A=\left(\begin{array}{cccc}
1 & 4 & 4^{2} & \ldots \\
a_{1} & a_{2} & a_{3} & \ldots \\
a_{2} & a_{3} & a_{4} & \cdots \\
\vdots & \vdots & \vdots & \ddots
\end{array}\right), \quad A^{\prime}=\left(\begin{array}{cccc}
1 & 4 & 4^{2} & \ldots \\
a_{2} & a_{3} & a_{4} & \ldots \\
a_{3} & a_{4} & a_{5} & \ldots \\
\vdots & \vdots & \vdots & \ddots
\end{array}\right)
$$

Note that the first row involves the powers of 4 and that $A^{\prime}$ corresponds to the shifted sequence $\left\{a_{2}, a_{3}, \ldots\right\}$. Let $A_{n}$ (resp. $A_{n}^{\prime}$ ) be the square matrix of order $n+1$ in the upper left-hand corner of $A$ (resp. $A^{\prime}$ ) so that, for example:

$$
A_{1}=\left(\begin{array}{cc}
1 & 4 \\
a_{1} & a_{2}
\end{array}\right), \quad A_{2}=\left(\begin{array}{ccc}
1 & 4 & 4^{2} \\
a_{1} & a_{2} & a_{3} \\
a_{2} & a_{3} & a_{4}
\end{array}\right), \quad \ldots
$$

The following identities will be proved in the Appendix (Section 9). 
Lemma 24. For all $n \geq 1$ one has:

$$
\operatorname{det} A_{n}=(-1)^{n} \quad \text { and } \quad \operatorname{det} A_{n}^{\prime}=(-1)^{n}(n+1) .
$$

We also need the following lemma, which follows easily by induction using the recursive scheme defining the numbers $T_{n j}$ and $U_{n j}$ in (49), and for which we omit the proof.

Lemma 25. For all $n \geq 1$ one has:

$$
T_{n n}=U_{n, n+1}=-1, \quad T_{n 0}=(-1)^{n+1} 4 n, \quad U_{n 0}=(-1)^{n+1} 4(2 n-1) .
$$

Moreover $T_{n j} \neq 0$ only when $0 \leq j \leq n$ and $U_{n j} \neq 0$ only when $0 \leq j \leq n+1$.

We will prove, by induction on $n$, the following set of identities. Note that identities $(c .1)$ and (c.2) are precisely the statement we want to show (that is, Proposition 23).

$$
\left\{\begin{array}{l}
\sum_{j=0}^{n} 4^{j} T_{n j}=\sum_{j=0}^{n+1} 4^{j} U_{n j}=0 \\
\sum_{j=0}^{n} a_{k+j} T_{n j}=\sum_{j=0}^{n+1} a_{k+j} U_{n j}=0 \quad \text { for all } k=1, \ldots, n-1, \quad(b .1),(a .2),(b .2) \\
\sum_{j=0}^{n} a_{n+j} T_{n j}=1, \quad \sum_{j=0}^{n+1} a_{n+j} U_{n j}=3 .
\end{array}\right.
$$

Let us introduce a useful notation. We let $B_{n}$ be the $n \times(n+1)$ matrix obtained by deleting the last row from $A_{n}$, and $B_{n}^{(j)}$ the $n \times n$ matrix obtained by deleting the $(j+1)$-th column from $B_{n}$ (that is, the column containing $4^{j}$ ). Then, in particular:

$$
B_{n}^{(n)}=A_{n-1} \text {. }
$$

For example:

$$
A_{2}=\left(\begin{array}{ccc}
1 & 4 & 4^{2} \\
a_{1} & a_{2} & a_{3} \\
a_{2} & a_{3} & a_{4}
\end{array}\right), B_{2}=\left(\begin{array}{ccc}
1 & 4 & 4^{2} \\
a_{1} & a_{2} & a_{3}
\end{array}\right)
$$

and

$$
B_{2}^{(0)}=\left(\begin{array}{cc}
4 & 4^{2} \\
a_{2} & a_{3}
\end{array}\right), \quad B_{2}^{(1)}=\left(\begin{array}{cc}
1 & 4^{2} \\
a_{1} & a_{3}
\end{array}\right), \quad B_{2}^{(2)}=\left(\begin{array}{cc}
1 & 4 \\
a_{1} & a_{2}
\end{array}\right)=A_{1} .
$$

Laplace rule applied to the last row of $A_{n}$ gives, since $\operatorname{det} A_{n}=(-1)^{n}$ :

$$
\sum_{j=0}^{n}(-1)^{j} a_{n+j} \operatorname{det} B_{n}^{(j)}=1
$$


Next, define the following vectors (in $\mathbf{R}^{n+1}$ and $\mathbf{R}^{n+2}$ respectively):

$$
\vec{T}_{n}=\left(\begin{array}{c}
T_{n 0} \\
T_{n 1} \\
\vdots \\
T_{n n}
\end{array}\right), \quad \vec{U}_{n}=\left(\begin{array}{c}
U_{n 0} \\
U_{n 1} \\
\vdots \\
U_{n, n+1}
\end{array}\right)
$$

It is clear that the identities (a.1), ., (c.2) are equivalent in vectorial form to:

$$
A_{n} \vec{T}_{n}=\left(\begin{array}{c}
0 \\
\vdots \\
0 \\
1
\end{array}\right), \quad B_{n+1} \vec{U}_{n}=\left(\begin{array}{c}
0 \\
\vdots \\
0 \\
3
\end{array}\right)
$$

Then, let us prove (52) by induction on $n$. We first verify it for $n=1$. From the recursive scheme (49) we see that $\vec{T}_{1}=\left(\begin{array}{c}4 \\ -1\end{array}\right)$ and $\vec{U}_{1}=\left(\begin{array}{c}4 \\ 3 \\ -1\end{array}\right)$. Hence:

$$
A_{1} \vec{T}_{1}=\left(\begin{array}{ll}
1 & 4 \\
1 & 3
\end{array}\right)\left(\begin{array}{c}
4 \\
-1
\end{array}\right)=\left(\begin{array}{l}
0 \\
1
\end{array}\right), \quad B_{2} \vec{U}_{1}=\left(\begin{array}{lll}
1 & 4 & 16 \\
1 & 3 & 10
\end{array}\right)\left(\begin{array}{c}
4 \\
3 \\
-1
\end{array}\right)=\left(\begin{array}{l}
0 \\
3
\end{array}\right) .
$$

which shows the assertion. As a double-check, we verify it also for $n=2$ :

$$
A_{2} \vec{T}_{2}=\left(\begin{array}{ccc}
1 & 4 & 16 \\
1 & 3 & 10 \\
3 & 10 & 35
\end{array}\right)\left(\begin{array}{c}
-8 \\
6 \\
-1
\end{array}\right)=\left(\begin{array}{l}
0 \\
0 \\
1
\end{array}\right), \quad B_{3} \vec{U}_{2}=\left(\begin{array}{cccc}
1 & 4 & 16 & 64 \\
1 & 3 & 10 & 35 \\
3 & 10 & 35 & 126
\end{array}\right)\left(\begin{array}{c}
-12 \\
-1 \\
5 \\
-1
\end{array}\right)=\left(\begin{array}{l}
0 \\
0 \\
3
\end{array}\right)
$$

We now assume that (52) is true for $n$ (equivalently, we assume the identities (a.1),..., (c.2) above) and prove it for $n+1$, that is, we must prove that

$$
\left\{\begin{array}{l}
\sum_{j=0}^{n+1} 4^{j} T_{n+1, j}=\sum_{j=0}^{n+2} 4^{j} U_{n+1, j}=0 \\
\sum_{j=0}^{n+1} a_{k+j} T_{n+1, j}=\sum_{j=0}^{n+2} a_{k+j} U_{n+1, j}=0 \quad \text { for all } k=1, \ldots, n \\
\sum_{j=0}^{n+1} a_{n+1+j} T_{n+1, j}=1, \quad \sum_{j=0}^{n+2} a_{n+1+j} U_{n+1, j}=3
\end{array}\right.
$$

We prove (1.1), (2.1), (3.1), (1.2), (2.2), (3.2) in that order. We recall from Lemma 25 that $T_{n j} \neq 0$ only when $0 \leq j \leq n$ and $U_{n j} \neq 0$ only when $0 \leq j \leq n+1$. 
Proof of (1.1). It follows from (a.1), (a.2) and the first equation in the scheme (49): $T_{n+1, j}=-3 T_{n j}+U_{n j}$.

Proof of (2.1). For $k=1, \ldots, n-1$ follows from the first equation in the scheme (49), (b.1), (b.2). For $k=n$ it follows from (c.1), (c.2).

Proof of (3.1). From (1.1) and (2.1) we see that $B_{n+1} \vec{T}_{n+1}=0$. The vector $\vec{T}_{n+1}$ is then a solution of a homogeneous system of $n+1$ equations in $n+2$ unknowns with coefficient matrix $B_{n+1}$. Therefore, there exists $\lambda \in \mathbf{R}$ such that

$$
T_{n+1, j}=(-1)^{j} \lambda \operatorname{det} B_{n+1}^{(j)}
$$

for all $j=0, \ldots, n+1$. We know from Lemma 25 that $T_{n+1, n+1}=-1$ and from Lemma 24 that $\operatorname{det} A_{n}=(-1)^{n}$. Therefore:

$$
\begin{aligned}
-1 & =T_{n+1, n+1} \\
& =(-1)^{n+1} \lambda \operatorname{det} B_{n+1}^{(n+1)} \\
& =(-1)^{n+1} \lambda \operatorname{det} A_{n} \\
& =(-1)^{n+1}(-1)^{n} \lambda \\
& =-\lambda
\end{aligned}
$$

hence $\lambda=1$ and, for all $j$ :

$$
T_{n+1, j}=(-1)^{j} \operatorname{det} B_{n+1}^{(j)} .
$$

On the other hand we know from (51) that for all $k$ one has $\sum_{j=0}^{k}(-1)^{j} a_{k+j} \operatorname{det} B_{k}^{(j)}=1$ hence, taking $k=n+1$ :

$$
\sum_{j=0}^{n+1} a_{n+1+j} T_{n+1, j}=\sum_{j=0}^{n+1}(-1)^{j} a_{n+1+j} \operatorname{det} B_{n+1}^{(j)}=1
$$

and (3.1) follows.

Proof of (1.2). One has from the second relation in scheme (49):

$$
\sum_{j=0}^{n+2} 4^{j} U_{n+1, j}=-4 \sum_{j=0}^{n+2} 4^{j} T_{n j}+\sum_{j=0}^{n+2} 4^{j} U_{n j}+\sum_{j=0}^{n+2} 4^{j} T_{n+1, j-1}
$$

The first two terms are zero by (a.1) and (a.2), the third equals $4 \sum_{j=0}^{n+2} 4^{j-1} T_{n+1, j-1}=$ $4 \sum_{i=0}^{n+1} 4^{i} T_{n+1, i}=0$ by $(1.1)$.

Proof of (2.2) One has from the second relation in scheme (49):

$$
\sum_{j=0}^{n+2} a_{k+j} U_{n+1, j}=-4 \sum_{j=0}^{n+2} a_{k+j} T_{n j}+\sum_{j=0}^{n+2} a_{k+j} U_{n j}+\sum_{j=0}^{n+2} a_{k+j} T_{n+1, j-1}
$$


If $k \leq n-1$ the first two terms on the right are zero by (b.1) and (b.2), and then:

$$
\sum_{j=0}^{n+2} a_{k+j} U_{n+1, j}=\sum_{j=0}^{n+2} a_{k+j} T_{n+1, j-1}=\sum_{i=0}^{n+1} a_{k+i+1} T_{n+1, i}=0
$$

because of (2.1). If $k=n$ the right-hand side equals

$$
\begin{aligned}
& -4 \sum_{j=0}^{n+2} a_{n+j} T_{n j}+\sum_{j=0}^{n+2} a_{n+j} U_{n j}+\sum_{j=0}^{n+2} a_{n+j} T_{n+1, j-1} \\
& =-4 \sum_{j=0}^{n} a_{n+j} T_{n j}+\sum_{j=0}^{n+1} a_{n+j} U_{n j}+\sum_{i=0}^{n+1} a_{n+1+i} T_{n+1, i} \\
& =-4+3+1 \\
& =0
\end{aligned}
$$

by (c.1), (c.2) and (3.1).

\section{Proof of (3.2) Set}

$$
\sum_{j=0}^{n+2} a_{n+1+j} U_{n+1, j}=\lambda .
$$

We have to show that $\lambda=3$. As $T_{n+2, j}=-3 T_{n+1, j}+U_{n+1, j}$ we see that, from (1.1) and (1.2), we have $\sum_{j} 4^{j} T_{n+2, j}=0$ and

$$
\sum_{j=0}^{n+2} a_{k+j} T_{n+2, j}=-3 \sum_{j=0}^{n+1} a_{k+j} T_{n+1, j}+\sum_{j=0}^{n+2} a_{k+j} U_{n+1, j} .
$$

If $k \leq n$ this is zero by (2.1) and (2.2). When $k=n+1$ this is $\lambda-3$ by (3.1) and (53). Expressed in matrix form, all this becomes the statement:

$$
B_{n+2} \vec{T}_{n+2}=\left(\begin{array}{c}
0 \\
\vdots \\
0 \\
\lambda-3
\end{array}\right)
$$

As $T_{n+2, n+2}=-1$ and $B_{n+2}^{(n+2)}=A_{n+1}$, taking the terms involving $T_{n+2, n+2}$ to the righthand side we see that (54) can be written:

$$
A_{n+1}\left(\begin{array}{c}
T_{n+2,0} \\
T_{n+2,1} \\
\vdots \\
T_{n+2, n+1}
\end{array}\right)=\left(\begin{array}{c}
4^{n+2} \\
a_{n+3} \\
\vdots \\
a_{2 n+3}+\lambda-3
\end{array}\right)
$$


By Cramer's rule, $T_{n+2,0}$ is the ratio:

$$
T_{n+2,0}=\frac{\operatorname{det} C_{n+2}}{\operatorname{det} A_{n+1}},
$$

where $C_{n+2}$ is the $(n+2) \times(n+2)$ matrix obtained replacing the first column of $A_{n+1}$ by the column

$$
\left(\begin{array}{c}
4^{n+2} \\
a_{n+3} \\
\vdots \\
a_{2 n+3}+\lambda-3
\end{array}\right)=\left(\begin{array}{c}
4^{n+2} \\
a_{n+3} \\
\vdots \\
a_{2 n+3}
\end{array}\right)+\left(\begin{array}{c}
0 \\
0 \\
\vdots \\
\lambda-3
\end{array}\right)
$$

Accordingly:

$$
\operatorname{det} C_{n+2}=\operatorname{det}\left(\begin{array}{cccc}
4^{n+2} & 4 & \ldots & 4^{n+1} \\
a_{n+3} & a_{2} & \ldots & a_{n+2} \\
\ldots & \ldots & \ldots & \ldots \\
a_{2 n+3} & a_{n+2} & \ldots & a_{2 n+2}
\end{array}\right)+\operatorname{det}\left(\begin{array}{cccc}
0 & 4 & \ldots & 4^{n+1} \\
0 & a_{2} & \ldots & a_{n+2} \\
\ldots & \ldots & \ldots & \ldots \\
\lambda-3 & a_{n+2} & \ldots & a_{2 n+2}
\end{array}\right) .
$$

Reordering columns and taking the factor 4 out of the determinants, we see:

$$
\operatorname{det} C_{n+2}=(-1)^{n+1} 4 \operatorname{det} A_{n+1}^{\prime}+(-1)^{n+1} 4(\lambda-3) \operatorname{det} A_{n}^{\prime} .
$$

We know from Lemma 24 that

$$
\operatorname{det} A_{n+1}^{\prime}=(-1)^{n+1}(n+2), \quad \operatorname{det} A_{n}^{\prime}=(-1)^{n}(n+1) .
$$

hence

$$
\operatorname{det} C_{n+2}=4(n+2)-4(\lambda-3)(n+1) \text {. }
$$

On the other hand, by (55) and Lemma 25, as $T_{n+2,0}=(-1)^{n+3} 4(n+2)$ :

$$
\begin{aligned}
\operatorname{det} C_{n+2} & =\left(\operatorname{det} A_{n+1}\right) T_{n+2,0} \\
& =4(n+2)
\end{aligned}
$$

Comparing these two last expressions we indeed get $\lambda-3=0$ hence $\lambda=3$. With this, the proof is complete.

\section{Appendix: proof of Lemma 24}

This section is based on the paper by Ulrich Tamm [26], and we will follow closely the notation there.

Given a sequence $\left\{c_{0}, c_{1}, c_{2}, \ldots\right\}$ form the infinite matrix

$$
A=\left(\begin{array}{cccc}
c_{0} & c_{1} & c_{2} & \cdots \\
c_{1} & c_{2} & c_{3} & \cdots \\
c_{2} & c_{3} & c_{4} & \cdots \\
\vdots & \vdots & \vdots & \ddots
\end{array}\right)
$$


and let $A_{n}$ be the $n \times n$ sub-matrix in the upper left corner:

$$
A_{1}=\left(c_{0}\right), \quad A_{2}=\left(\begin{array}{cc}
c_{0} & c_{1} \\
c_{1} & c_{2}
\end{array}\right), \quad A_{3}=\left(\begin{array}{lll}
c_{0} & c_{1} & c_{2} \\
c_{1} & c_{2} & c_{3} \\
c_{2} & c_{3} & c_{4}
\end{array}\right), \ldots
$$

In this way we obtain a sequence of Hankel matrices. We will also consider shifted sequences, namely for all $k \geq 0$ we set

$$
A^{(k)}=\left(\begin{array}{cccc}
c_{k} & c_{k+1} & c_{k+2} & \cdots \\
c_{k+1} & c_{k+2} & c_{k+3} & \cdots \\
c_{k+2} & c_{k+3} & c_{k+4} & \cdots \\
\vdots & \vdots & \vdots & \ddots
\end{array}\right)
$$

and let $A_{n}^{(k)}$ be the $n \times n$ sub-matrix in the upper left corner of $A^{(k)}$; by convention $A^{(0)}=A$. For all $k=0,1, \ldots$ we set

$$
d_{n}^{(k)}=\operatorname{det} A_{n}^{(k)} .
$$

By definition, the sequence $\left\{d_{1}^{(k)}, d_{2}^{(k)}, d_{3}^{(k)}, \ldots\right\}$ is called the Hankel transform of the sequence $\left\{c_{k}, c_{k+1}, c_{k+2} \ldots\right\}$.

Our interest is in the sequence

$$
c_{m}=\left(\begin{array}{c}
2 m+1 \\
m
\end{array}\right), \quad m \geq 0
$$

It is just the sequence defined in (45) with offset at $m=0$ :

$$
c_{m}=a_{m+1}=\{1,3,10,35,126,462,1716,6735, \ldots\} .
$$

Then

$$
A^{(0)}=\left(\begin{array}{ccccc}
1 & 3 & 10 & 35 & \cdots \\
3 & 10 & 35 & 126 & \cdots \\
10 & 35 & 126 & 462 & \cdots \\
\vdots & \vdots & \vdots & \vdots & \ddots
\end{array}\right), \quad \text { and } \quad A^{(1)}=\left(\begin{array}{ccccc}
3 & 10 & 35 & 126 & \cdots \\
10 & 35 & 126 & 462 & \cdots \\
35 & 126 & 462 & 1716 & \cdots \\
\vdots & \vdots & \vdots & \vdots & \ddots
\end{array}\right) .
$$

From part b) of Proposition 2.1 in [26] we see that, for all $n$ :

$$
d_{n}^{(0)}=1, \quad d_{n}^{(1)}=2 n+1,
$$

and the following expression holds for $k \geq 2$ :

$$
d_{n}^{(k)}=\Pi_{1 \leq i \leq j \leq k} \frac{i+j-1+2 n}{i+j-1} .
$$


Now introduce an indeterminate $x$ and consider the infinite matrices

$$
A=\left(\begin{array}{cccc}
1 & x & x^{2} & \cdots \\
c_{0} & c_{1} & c_{2} & \cdots \\
c_{1} & c_{2} & c_{3} & \cdots \\
c_{2} & c_{3} & c_{4} & \cdots \\
\vdots & \vdots & \vdots & \ddots
\end{array}\right), \quad A^{(1)}=\left(\begin{array}{cccc}
1 & x & x^{2} & \cdots \\
c_{1} & c_{2} & c_{3} & \cdots \\
c_{2} & c_{3} & c_{4} & \cdots \\
c_{3} & c_{4} & c_{5} & \cdots \\
\vdots & \vdots & \vdots & \ddots
\end{array}\right)
$$

and the sequences of polynomials $\left\{P_{n}(x)\right\},\left\{P_{n}^{(1)}(x)\right\}$ defined by

$$
\left\{\begin{array}{l}
P_{0}(x)=1, \quad P_{1}(x)=\left|\begin{array}{cc}
1 & x \\
c_{0} & c_{1}
\end{array}\right|, \quad P_{2}(x)=\left|\begin{array}{ccc}
1 & x & x^{2} \\
c_{0} & c_{1} & c_{2} \\
c_{1} & c_{2} & c_{3}
\end{array}\right|, \quad \ldots \\
P_{0}^{(1)}(x)=1, \quad P_{1}^{(1)}(x)=\left|\begin{array}{cc}
1 & x \\
c_{1} & c_{2}
\end{array}\right|, \quad P_{2}^{(1)}(x)=\left|\begin{array}{ccc}
1 & x & x^{2} \\
c_{1} & c_{2} & c_{3} \\
c_{2} & c_{3} & c_{4}
\end{array}\right|, \quad \ldots
\end{array}\right.
$$

Recalling that $c_{m}=a_{m+1}$ we see that proving Lemma 24 amounts to prove that, for all $n$ :

$$
P_{n}(4)=(-1)^{n}, \quad P_{n}^{(1)}(4)=(-1)^{n}(n+1) .
$$

Note that, by (56) , the leading coefficient of $P_{n}(x)$ (resp. $\left.P_{n}^{(1)}(x)\right)$ is $(-1)^{n} d_{n}=(-1)^{n}$ (resp. $\left.(-1)^{n} d_{n}^{(1)}=(-1)^{n}(2 n+1)\right)$. Then, the polynomials

$$
t_{n}(x)=(-1)^{n} P_{n}(x), \quad t_{n}^{(1)}(x)=\frac{(-1)^{n}}{2 n+1} P_{n}^{(1)}(x)
$$

are of degree $n$ and monic. In combinatorics, the sequence $\left\{t_{n}(x)\right\}$ is called the sequence of orthogonal polynomials associated to $\left\{c_{0}, c_{1}, \ldots\right\}$ (they coincide with the polynomials defined in (1.8) in [26]).

Taking into account (57) and (58), to prove Lemma 24 it is then sufficient to show the following fact.

Lemma 26. For the polynomials defined in (158) one has, for all $n$ :

$$
t_{n}(4)=1, \quad t_{n}^{(1)}(4)=\frac{n+1}{2 n+1} .
$$

Proof. It turns out that $\left\{t_{n}(x)\right\}$ and $\left\{t_{n}^{(1)}(x)\right\}$ satisfy a three-term recursive relation:

$$
\left\{\begin{array}{l}
t_{n}(x)=\left(x-\alpha_{n}\right) t_{n-1}(x)-\beta_{n-1} t_{n-2}(x), \quad t_{0}(x)=1, t_{1}(x)=x-\alpha_{1} \\
t_{n}^{(1)}(x)=\left(x-\alpha_{n}^{(1)}\right) t_{n-1}^{(1)}(x)-\beta_{n-1}^{(1)} t_{n-2}^{(1)}(x) \quad t_{0}^{(1)}(x)=1, t_{1}^{(1)}(x)=x-\alpha_{1}^{(1)}
\end{array}\right.
$$

for suitable numerical sequences $\alpha_{n} \doteq \alpha_{n}^{(0)}, \beta_{n} \doteq \beta_{n}^{(0)}, \alpha_{n}^{(1)}, \beta_{n-1}^{(1)}$. This sequences have been computed in [26]. In fact, for arbitrary $k$, the coefficients $\alpha_{n}^{(k)}, \beta_{n-1}^{(k)}$ can be computed in 
terms of the coefficients $q_{n}^{(k)}, e_{n}^{(k)}$ in the continued fraction expansion of $1-x F(x)$, where $F(x)=\sum_{m=0}^{\infty} c_{m+k} x^{m}$ (see (1.14), (1.19) and (1.20) in [26]):

$$
\alpha_{1}=q_{1} \quad \text { and, for } n \geq 1: \quad \alpha_{n+1}^{(k)}=q_{n+1}^{(k)}+e_{n}^{(k)}, \quad \beta_{n}^{(k)}=q_{n}^{(k)} \cdot e_{n}^{(k)},
$$

For our sequence $c_{m}=\left(\begin{array}{c}2 m+1 \\ m\end{array}\right)$ the corresponding coefficients have been computed in Corollary 2.1, equation (2.6):

$$
q_{n}^{(k)}=\frac{(2 n+2 k)(2 n+2 k+1)}{(2 n+k-1)(2 n+k)}, \quad e_{n}^{(k)}=\frac{(2 n-1)(2 n)}{(2 n+k)(2 n+k+1)} .
$$

In particular,

$$
\left\{\begin{array} { l } 
{ q _ { n } = q _ { n } ^ { ( 0 ) } = \frac { 2 n + 1 } { 2 n - 1 } } \\
{ e _ { n } = e _ { n } ^ { ( 0 ) } = \frac { 2 n - 1 } { 2 n + 1 } }
\end{array} \text { and } \quad \left\{\begin{array}{l}
q_{n}^{(1)}=\frac{(2 n+2)(2 n+3)}{(2 n)(2 n+1)} \\
e_{n}^{(1)}=\frac{(2 n-1)(2 n)}{(2 n+1)(2 n+2)}
\end{array}\right.\right.
$$

From (60) we obtain $\alpha_{1}=q_{1}=3, \alpha_{1}^{(1)}=q_{1}^{(1)}=\frac{10}{3}$ and for $n \geq 2$ (after some calculations):

$$
\left\{\begin{array} { l } 
{ \alpha _ { n } = 2 } \\
{ \beta _ { n - 1 } = 1 }
\end{array} , \text { and } \left\{\begin{array}{l}
\alpha_{n}^{(1)}=2+\frac{4}{(2 n+1)(2 n-1)} \\
\beta_{n-1}^{(1)}=\frac{(2 n-3)(2 n+1)}{(2 n-1)^{2}}
\end{array}\right.\right.
$$

From (59) and (61) one has the following recursive scheme for the sequence $\left\{t_{n}(x)\right\}$ :

$$
\left\{\begin{array}{l}
t_{n}(x)=(x-2) t_{n-1}(x)-t_{n-2}(x) \\
t_{0}(x)=1 \\
t_{1}(x)=x-3
\end{array}\right.
$$

Setting $X_{n}=t_{n}(4)$ we obtain

$$
\left\{\begin{array}{l}
X_{n}=2 X_{n-1}-X_{n-2} \\
X_{0}=1 \\
X_{1}=1
\end{array}\right.
$$

hence $X_{n}=1$ for all $n$. That is:

$$
t_{n}(4)=1
$$

for all $n$. This shows the first relation in the Lemma.

About the sequence $\left\{t_{n}^{(1)}(x)\right\}$ we know:

$$
\left\{\begin{array}{l}
t_{n}^{(1)}(x)=\left(x-\alpha_{n}^{(1)}\right) t_{n-1}^{(1)}(x)-\beta_{n-1}^{(1)} t_{n-2}^{(1)}(x) \\
t_{0}^{(1)}(x)=1 \\
t_{1}^{(1)}(x)=x-\frac{10}{3}
\end{array}\right.
$$


where $\alpha_{n}^{(1)}$ and $\beta_{n-1}^{(1)}$ are as in (61). We are interested in the sequence $X_{n}=t_{n}^{(1)}(4)$, which obeys the recursive law:

$$
\left\{\begin{array}{l}
X_{n}=\left(4-\alpha_{n}^{(1)}\right) X_{n-1}-\beta_{n-1}^{(1)} X_{n-2} \\
X_{0}=1 \\
X_{1}=\frac{2}{3}
\end{array}\right.
$$

By induction on $n$, let us show that $X_{n}=\frac{n+1}{2 n+1}$. In fact this holds for $n=1$. Assume it to be true for all $k \leq n-1$. By (61):

$$
4-\alpha_{n}^{(1)}=\frac{8 n^{2}-6}{(2 n-1)(2 n+1)}, \quad \beta_{n-1}^{(1)}=\frac{(2 n-3)(2 n+1)}{(2 n-1)^{2}}
$$

hence:

$$
\begin{aligned}
X_{n} & =\left(4-\alpha_{n}^{(1)}\right) X_{n-1}-\beta_{n-1}^{(1)} X_{n-2} \\
& =\frac{8 n^{2}-6}{(2 n-1)(2 n+1)} \cdot \frac{n}{2 n-1}-\frac{(2 n-3)(2 n+1)}{(2 n-1)^{2}} \cdot \frac{n-1}{2 n-3} \\
& =\frac{n+1}{2 n+1}
\end{aligned}
$$

which shows the claim. With this, the proof of Lemma 24 is complete.

Remark. We point out that the expression of $\alpha_{n+1}^{(k)}$ in the statement of Corollary 2.3 in [26] is wrong; this is due to an incorrect algebraic manipulation of $q_{n+1}^{(k)}+e_{n}^{(k)}$ in the proof of Corollary 2.3 there. The correct value of $\alpha_{n+1}^{(1)}$ shown in (61) is directly computed from the expressions of $q_{n}^{(1)}$ and $e_{n}^{(1)}$ taken from Corollary 2.1, equation (2.6) of [26].

Acknowledgements. We wish to thank Sylvestre Gallot for his interest and for many enlightening discussions, and Stefano Capparelli for pointing out to us the role of orthogonal polynomials in the combinatorial part of the paper (Lemma 24).

\section{References}

[1] M. van den Berg and P. Gilkey Heat content asymptotics for a Riemannian manifold with boundary, J. Funct. Anal. 120 (1994), 48-71

[2] M. van den Berg and P. Gilkey The heat equation with inhomogeneous Dirichlet boundary conditions, Comm. Anal. Geom. 7, n. 2 (1999), 279-294

[3] C.A. Berenstein An inverse spectral theorem and its applications to the Pompeiu problem, J. Anal. Math. 37 (1980), 128-144 
[4] C.A. Berenstein On an overdetermined Neumann problem, Geometry Seminars (1986) 21-26, Univ. Stud. Bologna, Bologna 1988

[5] C. A. Berenstein and M. Shahshahani, Harmonic analysis and the Pompeiu problem, Amer. J. Math. 105 (1983), 1217-1229

[6] C. Berenstein and P. Yang, An inverse Neumann problem, J. Reine Angew. Math. 382 (1987), 1-21

[7] L. Caffarelli, The regularity of free boundaries in higher dimensions, Acta Math. 139 (1977), 155-184

[8] S. Capparelli and P. Maroscia, On two sequences of othogonal polynomials related to Jordan blocks, Mediterranean J. Math. 10 n. 4 (2013), 1609-1630

[9] E. Cartan, Familles de surfaces isoparamétriques dans les espaces à courbure constante, Annali di Mat. 17 (1938), 177-191

[10] L. Chakalov, Sur un probleme de D. Pompeiu, Annuaire Univ. Sofia Phys.-Math, Livre I, 40 (1944), 1-44

[11] A. El Soufi and S. Ilias Domain deformations and eigenvalues of the Dirichlet Laplacian in Riemannian manifolds, Illinois J. Math. 51 (2007) 645-666

[12] P.R. Garadedian and M. Schiffer, Variational problems in the theory of elliptic partial differential equations, J. Rational Mech. Anal. 2 (1953) 137-171

[13] D. Kinderleher and L. Nirenberg, Regularity in free boundary problems, Ann. Scuola Norm. Sup. Pisa Cl. Sci. 4 (1977). 373-391

[14] S. Kumaresan and J. Prajapat, Serrin's result for hyperbolic space and sphere, Duke Math. J. 91 n.1 (1998), 17-28

[15] G. Liu, Symmetry theorems for the overdetermined eigenvalue problems, J. Diff. Eq. 233 (2007) 585-600

[16] R. Magnanini and S. Sakaguchi, Heat conductors with a stationary isothermic surface, Ann. Math. Second Series, 156 no. 3 (2002), 931-946

[17] R. Molzon, Symmetry and overdetermined boundary problems, Forum Math. 3 (1991), 143-156

[18] H.F. Münzner, Isoparametrische hyperflachen in spharen I, II, Math. Ann. 251 (1980), 57-71, and 256 (1981), 215-232

[19] F. Pacard and P. Sicbaldi, Extremal domains for the first eigenvalue of the LaplaceBeltrami operator, Ann. Institut Fourier 59 No. 2 (2009), 515-542 
[20] S. Raulot and A. Savo On the first eigenvalue of the Dirichlet-to-Neumann operator on forms J. Funct. Anal. 262 (2012), 889-914

[21] A. Savo, Uniform estimates and the whole asymptotic series of the heat content on manifolds, Geom. Dedicata 73 (1998), 181-214

[22] A. Savo, Asymptotics of the heat flow for domains with smooth boundary, Comm. Anal. Geom. 12 No. 3 (2004), 671-702

[23] M. Schiffer, Hadamard's formula and variation of domain functions, Amer. J. Math. 68 (1946), 417-448

[24] J. Serrin, A symmetry problem in potential theory, Arch. Rational Mech. Anal. 43 (1971), 304-318

[25] V. Shklover, Schiffer problem and isoparametric hypersurfaces, Revista Mat. Iberoamericana 16 n. 3 (2000), 529-569

[26] U. Tamm, Some aspects of Hankel matrices in Coding Theory and Combinatorics, The Electronic Journal of Combinatorics 8 (2001) \# A1

[27] G. Thorbergsson, A survey on isoparametric hypersurfaces and their generalizations, Handbook of Differential Geometry, Vol. 1, 963-995, North-Holland Amsterdam 2000

[28] Q.M. Wang, Isoparametric functions on Riemannian manifolds, I, Math. Ann. 277 (1987), 639-646

[29] H.F. Weinberger, Remark on the preceeding paper by Serrin, Arch. Rational Mech. Anal. 43 (1971), 319-320

[30] S. A. Williams, A partial solution of the Pompeiu problem, Math. Ann. 223 (1976), 183-190

[31] S. A. Williams, Analyticity of the boundary for Lipschitz domains without the Pompeiu property, Indiana Univ. Math. J. 30 (1981), 357-369

Author address:

Alessandro Savo

Dipartimento SBAI, Sezione di Matematica

Sapienza Università di Roma

Via Antonio Scarpa 16

00161 Roma, Italy

E-Mail: alessandro.savo@sbai.uniroma1.it 